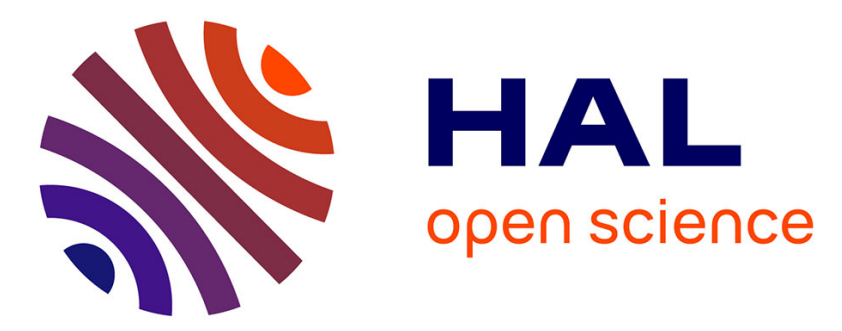

\title{
Mistuning analysis and uncertainty quantification of an industrial bladed disk with geometrical nonlinearity
}

Evangéline Capiez-Lernout, Christian Soize, Moustapha Mbaye

\section{To cite this version:}

Evangéline Capiez-Lernout, Christian Soize, Moustapha Mbaye. Mistuning analysis and uncertainty quantification of an industrial bladed disk with geometrical nonlinearity. Journal of Sound and Vibration, 2015, 356, pp.124-143. 10.1016/j.jsv.2015.07.006 . hal-01183415

\section{HAL Id: hal-01183415 https://hal.science/hal-01183415}

Submitted on 7 Aug 2015

HAL is a multi-disciplinary open access archive for the deposit and dissemination of scientific research documents, whether they are published or not. The documents may come from teaching and research institutions in France or abroad, or from public or private research centers.
L'archive ouverte pluridisciplinaire HAL, est destinée au dépôt et à la diffusion de documents scientifiques de niveau recherche, publiés ou non, émanant des établissements d'enseignement et de recherche français ou étrangers, des laboratoires publics ou privés. 
E. Capiez-Lernout ${ }^{1, a, \star}$, C. Soize ${ }^{2, a}$, M. Mbaye ${ }^{3, b}$

${ }^{1}$ Evangeline.Capiez-Lernout@univ-paris-est.fr

${ }^{2}$ Christian.Soize@univ-paris-est.fr

${ }^{3}$ Moustapha.Mbaye@turbomeca.fr

${ }^{a}$ Université Paris-Est

Laboratoire Modélisation et Simulation Multi-Echelle MSME UMR 8208 CNRS

5, Boulevard Descartes,

F-77454 Marne-La-Vallée Cedex 02, France

Tel: 0033160957798

Fax: 0033160957799.

${ }^{b}$ Turbomeca SAFRAN Group

64511 Bordes, France.

* Corresponding author. 


\title{
Mistuning analysis and uncertainty quantification of an industrial bladed disk with geometrical nonlinearity.
}

\begin{abstract}
This paper deals with the dynamical analysis and uncertainty quantification of a mistuned industrial rotating integrally bladed disk, for which the operating regime under consideration takes into account the nonlinear geometrical effects induced by large displacements and deformations. First, a dedicated mean nonlinear reduced-order model of the tuned structure is explicitly constructed using the finite element method. The random nature of the mistuning is then modeled by using the nonparametric probabilistic approach extended to the nonlinear geometric context. Secondly, a detailed dynamic analysis and uncertainty propagation are conducted in order to quantify the impact of the nonlinear geometrical effects on the mistuned structure. The results show that the dynamic amplification in the frequency band is significant outside the frequency band of excitation due to the presence of geometric nonlinearities combined with mistuning effects.
\end{abstract}

Keywords:

Mistuning, Bladed-disk, Geometric nonlinearities, Uncertainty quantification

\section{Introduction}

This paper proposes a detailed dynamic analysis of industrial rotating integrally bladed disks with respect to the nonlinear geometrical effects in presence of an uncertain mistuning. Very few works have been published in this particular field that seems more and more important for analyzing exceptional operating regime in turbomachinery. In the framework of linear mistuning analysis, amplifications of the response are observed in the frequency band of excitation while in presence of geometric nonlinearities, there is a transfer of vibrational energy outside the frequency band of excitation with complex uncertainty propagation mechanisms. Hereinafter, we begin this introduction with a brief history concerning the mistuning and nonlinearities effects in structural dynamics and in turbomachinery.

A main challenge in structural dynamics is the development of advanced numerical methodologies for the construction of robust computational models in order to efficiently predict the 
dynamical behavior of structures. The present manuscript investigates, more particularly, the aeronautical area for which the mistuning of turbomachinery-bladed disks has to be taken into account in the computational models. It is well known that the natural cyclic symmetry of such structures is broken due to manufacturing tolerances and material dispersions, which create small discrepancies from one blade to another one. Such phenomena, referred to mistuning, can generate localization effects combined to a dynamic amplification of the forced response [1]. Many research efforts have been carried out on this subject, including the construction of reduced-order models with probabilistic approaches for taking into account the random character of the mistuning in the computational models (see e.g. [2, 3]). For the robust design of such mistuned structures, different strategies have then been considered (see e.g. $[4,5,6,7,8]$ ). Most of these works have been carried out in a linear context.

Nevertheless, another essential aspect is to pay a particular attention to the various nonlinear effects that need to be taken into account in the computational models. It should be underlined that large research efforts have been made in order to include the local nonlinearities such as nonlinear contact interfaces $[9,10,11]$. Furthermore, given the constant growing of the computational capabilities, including the possibility of using parallel computations, it seems quite appropriate to consider the geometrically nonlinear effects in the computational models $[12,13]$. Indeed, the recent improvements in turbomachinery design, combined to the unavoidable requirements of kerosene savings, require to analyze the exceptional operating regime of bladed disks, for which large deformations can occur $[14,15,16,17]$. It corresponds, for instance, to nearly unstable situations induced by the aerodynamic coupling that can lead flutter and thus inducing very low damping levels. In a way, such situation is equivalent to the case of severe loading and the usual linearized elastodynamic theory cannot be used any more. In such a case, the geometrically nonlinear effects induced by the large displacements/deformations cannot be ignored and need to be taken into account in the computational models.

A special attention has to be first given to the case of geometrically nonlinear tuned bladed disks, which have the perfect symmetry. The large set of nonlinear coupled differential equations issued from the computational model of the tuned structure has to necessarily be solved in the time domain, leading for introducing a reduced-order model. This requires first the selection of an appropriate deterministic basis for constructing the representation of the nonlinear 
dynamical response. Such basis could be obtained by the Proper Orthogonal Decomposition method [18], known to be particularly efficient for nonlinear static cases (see e.g. [19] and included references) and that requires a particular attention in nonlinear structural dynamics, or by using appropriately selected natural linear elastic modes of vibration (see the review presented in [20]). The operators of the corresponding nonlinear reduced-order model can then be deduced from the STEP method [12,13] or from an explicit construction as proposed in [21] using three-dimensional solid finite elements or proposed in [22] using shell finite elements. One also have to focus on the modeling of the external load, corresponding to a chosen frequency band of excitation, which has to be selected according to usual turbomachinery criterions. The external load has to be defined in the time domain and must represent a uniform sweep in the frequency domain. After having established the mean nonlinear reduced-order model (MEANNL-ROM) that allows the geometrically nonlinear dynamical analysis of the tuned structure to be performed, we propose to implement the mistuning uncertainties using the nonparametric probabilistic framework [23]. The uncertainty induced by mistuning is implemented for each operator. The mistuning level is then controlled by a limited number of hyperparameters controlling the amount of uncertainties for each random operator. A stochastic reduced-order model (STOCH-NL-ROM) is then obtained, which leads for solving a reasonable number of uncertain nonlinear coupled differential equations in the time domain, requiring the construction of efficient and dedicated algorithms.

In this paper, such a computational strategy provides an efficient tool, which is applied to a computational model of an industrial centrifugal compressor with a large number of degrees of freedom. This allows for putting in evidence some new complex dynamical behaviors. Indeed, some complex mechanisms can be observed for the energy transfer through both geometric nonlinearities and mistuning uncertainties outside the frequency band of excitation. Different criterions are constructed (1) for detecting the threshold from which geometric nonlinear effects arise and (2) for quantifying the energy transfer that can occur in the frequency band. A careful and detailed analysis is carried out because unexpected dangerous situations, which can alter the life duration of the bladed disks, can potentially exist.

The paper is organized as follows. Section 1 summarizes a complete description of the computational methodology and of its numerical aspects allowing the nonlinear dynamic analy- 
sis of mistuned bladed disks to be done. The MEAN-NL-ROM corresponding to the tuned structure is explicitly constructed following the approach presented in [21]. Once the uncertainties induced by mistuning are modeled through the nonparametric probabilistic approach, the numerical strategy is discussed for the resolution of the STOCH-NL-ROM. Section 2 that constitutes the main objective of this paper, is devoted to a detailed dynamic analysis and to uncertainty propagation in order to quantify the impact of the nonlinear geometrical effects on an industrial centrifugal compressor. The nonlinear tuned dynamic analysis is investigated in both time domain and frequency domain. A sensitivity analysis is conducted with respect to the load intensity (amplitude) in order to quantify the effects of the geometric nonlinearities. In addition, a nonlinear sensitivity analysis is carried out with respect to the different types of uncertainties sources induced by the mistuning, in order to qualify and to quantify the different mistuning effects that can occur in presence of geometric nonlinearities. Finally, a complete nonlinear dynamical analysis of the mistuned response is carried out and the confidence region of the response amplifications are compared between the linear and nonlinear cases on the whole frequency band of analysis.

\section{Methodology}

This Section is devoted to the construction of a methodology for the nonlinear mistuning analysis occurring in rotating bladed-disks structures. In the present work, the bladed disk under consideration is assumed (1) to be made up of a linear elastic material and (2) to undergo large displacements and large deformations inducing geometrical nonlinearities.

\subsection{Description of the geometric nonlinear boundary value problem}

The structure under consideration is a bladed-disk structure with a $M$-order cyclic symmetry. Thus, the geometrical domain, the material constitutive equation, and the boundary conditions related to the generating sector are invariant under the $2 \pi / M$ rotation around its axis of symmetry. Moreover, the bladed disk undergoes a rotational motion around the axis of symmetry with constant angular speed $\Omega$. The structure is made up of a linear elastic material and is assumed to undergo large deformations inducing geometrical nonlinearities. A total Lagrangian formulation is chosen, which means that the dynamical equations are expressed in the rotating 
frame of an equilibrium configuration considered as a natural configuration. Let $\Omega$ be the threedimensional bounded domain corresponding to such reference configuration and subjected to the body force field $\mathbf{g}(\mathbf{x}, t)$, in which $\mathbf{x}$ denotes the position of a given point belonging to domain $\boldsymbol{\Omega}$. The boundary $\partial \boldsymbol{\Omega}$ is such that $\partial \boldsymbol{\Omega}=\boldsymbol{\Gamma} \cup \boldsymbol{\Sigma}$ with $\boldsymbol{\Gamma} \cap \boldsymbol{\Sigma}=\emptyset$ and the external unit normal to boundary $\partial \Omega$ is denoted by $\mathbf{n}$. The boundary part $\Gamma$ corresponds to the fixed part of the structure (in the local rotating frame $\left(\mathbf{x}_{1}, \mathbf{x}_{2}, \mathbf{x}_{3}\right)$ ) whereas the boundary part $\Sigma$ is subjected to the external surface force field $\mathbf{G}(\mathbf{x}, t)$. Note that the external force fields are derived from the Lagrangian transport into the reference configuration of the physical body/surface force fields applied in the deformed configuration. The external load can represent, for instance, the unsteady pressures applied to the blades. The displacement field expressed with respect to the reference configuration is denoted as $\mathbf{u}(\mathbf{x}, t)$.

\subsection{Construction of the MEAN-NL-ROM related to the tuned structure}

Let $\mathcal{C}$ be the admissible space defined by

$$
\mathcal{C}=\{\mathbf{v} \in \Omega, \mathbf{v} \text { sufficiently regular }, \mathbf{v}=\mathbf{0} \text { on } \Gamma\}
$$

The vector $\mathbf{q}=\left(q_{1}, \ldots, q_{N}\right)$ of the generalized coordinates is then introduced as a new set of unknown variables by projecting the reference nonlinear response $\mathbf{u}(\mathbf{x}, \cdot)$ on the vector space spanned by the finite family $\left.\varphi^{1}, \cdots, \varphi^{N}\right\}$ of a given vector basis of $\mathcal{C}$. The MEAN-NL-ROM is then described by the approximation $\mathbf{u}^{N}(\mathbf{x}, t)$ of order $N$ of $\mathbf{u}(\mathbf{x}, t)$ such that

$$
\mathbf{u}^{N}(\mathbf{x}, t)=\sum_{\beta=1}^{N} \boldsymbol{\varphi}^{\beta}(\mathbf{x}) q_{\beta}(t),
$$

in which $\mathbf{q}$ is solution of the nonlinear differential equation

$$
m_{\alpha \beta} \ddot{q}_{\beta}+\left(d_{\alpha \beta}+c(\Omega)_{\alpha \beta}\right) \dot{q}_{\beta}+\left(k_{\alpha \beta}^{(e)}+k^{(c)}(\Omega)_{\alpha \beta}+k_{\alpha \beta}^{(g)}\right) q_{\beta}+k_{\alpha \beta \gamma}^{(2)} q_{\beta} q_{\gamma}+k_{\alpha \beta \gamma \delta}^{(3)} q_{\beta} q_{\gamma} q_{\delta}=f_{\alpha}
$$

In all the paper, the usual convention of summation over Greek or Latin repeated indices is used. Let $\rho(\mathbf{x})$ be the mass density field expressed in the reference configuration and let $\mathbb{a}=\left\{a_{i j k \ell}\right\}_{i j k \ell}$ be the fourth-order elasticity tensor, which satisfies the usual symmetry, boundedness, and positive-definiteness properties. In Eq. (3), the reduced operators $[\mathbf{m}],\left[\mathbf{k}^{(g)}\right]$ and 
$\left[\mathbf{k}^{(e)}\right]$ are the mass, geometrical stiffness, and elastic stiffness real $(N \times N)$ matrices with positive-definiteness property, for which entries are defined by

$$
\begin{gathered}
m_{\alpha \beta}=\int_{\Omega} \rho \varphi_{i}^{\alpha} \varphi_{i}^{\beta} \mathrm{d} \mathbf{x} \\
k_{\alpha \beta}^{(e)}=\int_{\Omega} a_{j k \ell m} \varphi_{j, k}^{\alpha} \varphi_{\ell, m}^{\beta} \mathrm{d} \mathbf{x}, \\
k_{\alpha \beta}^{(g)}=\int_{\Omega} \sigma_{i j}^{(g)} \varphi_{s, i}^{\alpha} \varphi_{s, j}^{\beta} \mathrm{d} \mathbf{x}
\end{gathered}
$$

where the second-order symmetric tensor field $\sigma^{(g)}=\left\{\sigma_{i j}^{(g)}\right\}_{i j}$ represents the Cauchy constraints acting on the reference configuration, and where $\psi_{i, j}=\frac{\partial \psi_{i}}{\partial x_{j}}$. The rotational effects are taken into account through the reduced operators $[\mathbf{c}(\Omega)]$ and $\left[\mathbf{k}^{(c)}(\Omega)\right]$, which represent the gyroscopic coupling term with antisymmetry property and the centrifugal stiffness term with negative-definiteness property, which are written as

$$
\begin{aligned}
c_{\alpha \beta}(\Omega) & =\int_{\Omega} 2 \rho r_{i j} \varphi_{j}^{\alpha} \varphi_{i}^{\beta} \mathrm{d} \mathbf{x}, \\
k_{\alpha \beta}^{(c)}(\Omega) & =\int_{\Omega} \rho r_{i k} r_{k j} \varphi_{j}^{\alpha} \varphi_{i}^{\beta} \mathrm{d} \mathbf{x},
\end{aligned}
$$

where the $(3 \times 3)$ matrix $[\mathbf{r}]$ is such that $r_{i j}=-\Omega \varepsilon_{i j 3}$, where $\varepsilon_{i j k}$ is the Levi-Civita symbol such that $\varepsilon_{i j k}= \pm 1$ for an even or odd permutation and $\varepsilon_{i j k}=0$ otherwise. It should be noted that the centrifugal effects are assumed to be sufficiently small so that the linear stiffness reduced matrix $[\mathbf{k}(\Omega)]=\left[\mathbf{k}^{(e)}\right]+\left[\mathbf{k}^{(c)}(\Omega)\right]+\left[\mathbf{k}^{(g)}\right]$ is positive definite, yielding only stable dynamical systems to be considered. The geometric nonlinearities are taken into account through the quadratic and cubic stiffness contributions $k_{\alpha \beta \gamma}^{(2)}$ and $k_{\alpha \beta \gamma \delta}^{(3)}$ which are written as

$$
\begin{gathered}
k_{\alpha \beta \gamma}^{(2)}=\frac{1}{2}\left(\widehat{k}_{\alpha \beta \gamma}^{(2)}+\widehat{k}_{\beta \gamma \alpha}^{(2)}+\widehat{k}_{\gamma \alpha \beta}^{(2)}\right), \\
\widehat{k}_{\alpha \beta \gamma}^{(2)}=\int_{\Omega} a_{j k \ell m} \varphi_{j, k}^{\alpha} \varphi_{s, \ell}^{\beta} \varphi_{s, m}^{\gamma} \mathrm{d} \mathbf{x}, \\
k_{\alpha \beta \gamma \delta}^{(3)}=\frac{1}{2} \int_{\Omega} a_{j k \ell m} \varphi_{r, j}^{\alpha} \varphi_{r, k}^{\beta} \varphi_{s, \ell}^{\gamma} \varphi_{s, m}^{\delta} \mathrm{d} \mathbf{x} .
\end{gathered}
$$


It can easily be shown that tensor $k_{\alpha \beta \gamma}^{(2)}$ has permutation-invariance property and that tensor $k_{\alpha \beta \gamma \delta}^{(3)}$ has positive-definiteness property. Concerning the reduced damping operator, a modal damping model is added. Finally, the reduced external load is written as

$$
f_{\alpha}=\int_{\Omega} g_{i} \varphi_{i}^{\alpha} \mathrm{d} \mathbf{x}+\int_{\Sigma} G_{i} \varphi_{i}^{\alpha} \mathrm{d} \mathbf{s}+\int_{\Omega} \rho r_{i j} r_{j k}\left(x_{0}\right)_{k} \varphi_{k}^{\alpha} \mathrm{d} \mathbf{x}
$$

in which $x_{0}$ is the current position in the natural configuration [24].

Concerning the choice of the projection basis, the one related to the linear eigenvalue problem of the rotating tuned conservative structure, for which the gyroscopic coupling effects are ignored, is chosen. Since the tuned structure has a perfect cyclic symmetry, the use of the discrete Fourier transform allows for rewriting the eigenvalue problem of the whole tuned structure into uncoupled sub-eigenvalue problems related to the generator sector with appropriate boundary conditions $[25,26]$. The eigenvectors of the whole tuned structure are then reconstructed in its corresponding physical space. Note that these eigenvectors are ordered by increasing values of their corresponding eigenvalues $\lambda_{\alpha}, \alpha \in\{1, \ldots, N\}$ and verify the following orthogonality properties,

$$
m_{\alpha \beta}=\delta_{\alpha \beta} \quad, \quad k_{\alpha \beta}^{(g)}+k^{(c)}(\Omega)_{\alpha \beta}+k_{\alpha \beta}^{(e)}=\lambda_{\alpha} \delta_{\alpha \beta} \quad,
$$

where $\delta_{\alpha \beta}$ is the Kronecker symbol such that $\delta_{\alpha \beta}=1$ if $\alpha=\beta$ and $\delta_{\alpha \beta}=0$ otherwise. It should be noted that such projection basis issued from a linear eigenvalue problem is used for the construction of the MEAN-NL-ROM, which means that a systematic convergence analysis with respect to $N$ is carried out so that the MEAN-NL-ROM is representative of the nonlinear dynamical behavior of the structure.

\subsection{Numerical aspects for the construction of the nonlinear reduced operators}

There are many strategies, allowing a MEAN-NL-ROM to be established, depending on the choice of the vector basis (see for instance [20]) or the way to extract the reduced operators from explicit construction $[21,22]$ or from an implicit non intrusive construction $[12,20]$. In the present context, the MEAN-NL-ROM is explicitly constructed from the knowledge of the projection basis. It is carried out in the context of the three-dimensional finite element method, for which the finite elements are chosen as isoparametric solid finite elements with 8 nodes 
with a numerical integration using 8 Gauss integration points. Concerning the construction of the quadratic and cubic nonlinear stiffness operators, the methodology presented in [21] is used. In particular, due to the chosen strategy for the mistuning modeling, it should be underlined that the $\widehat{k}_{\alpha \beta \gamma}^{(2)}$ and $k_{\alpha \beta \gamma \delta}^{(3)}$ nonlinear stiffness entries have to be explicitly known. The numerical procedure uses the symmetry properties of the reduced operators, combined with the use of distributed calculations in parallel computer in order to optimize its efficiency. The main steps, which can be found in details in [21], require (1) the computation of the elementary contributions of each type of internal forces projected on the vector basis, (2) the finite element assembly of these elementary contributions, (3) the computation of the reduced operators by projecting each assembled internal force on the projection vector basis.

\subsection{Strategy for the construction of the external load}

In the present case, the presence of the geometric nonlinearities yields the nonlinear differential equation Eq. (3) to be solved in the time domain, the frequency content of the nonlinear dynamical response being a posteriori post-analyzed by using a Fast-Fourier-Transform (FFT). The reduced excitation issued from the external load is assumed to be splitted according to a spatial part and to a time-domain part such that

$$
f_{\alpha}(t)=f_{0} s_{\alpha} g(t)
$$

in which $f_{0}$ is a coefficient characterizing the global load intensity, where $\mathbf{s}$ is a $\mathbb{R}^{N}$-vector corresponding to the spatial modal contribution of the external load, and where $g(t)$ describes the time evolution of the load. Similarly to the usual linear analysis of structures with $M$-order cyclic symmetry, the excitation is constructed with a cyclic spatial repartition and a constant phase shift $(2 \pi h) / M$ from one blade to another one, so that only the eigenfrequencies corresponding to a given $h$ circumferential wave number are excited. Note that the use of the cyclic symmetry property has no real interest for expressing the nonlinear response according to its harmonic components because a decoupling between the harmonic components cannot be obtained. Moreover, it should be recalled that the usual linear analysis of bladed-disk structures requires to display the eigenfrequencies of the structure with respect to its circumferential wave number. It can be shown that the eigenmodes corresponding to localized blade modes are characterized by straight lines contrary to the eigenmodes corresponding to global coupled disk- 
blade modes. It is well known that the mistuning important effects of response amplification are concentrated in "veering" zones for which the coupling between the disk motion and the blade motion is high $[1,27,28,29]$. Indeed, in such veering zones, the tuned responses are known to be inhibited with respect to the ones corresponding to pure blade mode excitations, due to the repartition of the mechanical energy between disk and blade modes. This helps to understand why the mistuning effects yield so strong response amplifications [30]. For this reason, the nonlinear dynamical analysis has to be performed in a chosen frequency band of excitation and not for a single frequency excitation. Because of the geometric nonlinearities, the use of a harmonic excitation seems to be inappropriate because the set of nonlinear coupled differential equations should be solved for each harmonic excitation considered. The strategy is to simultaneously and uniformly excite all the frequencies of the given frequency band of excitation so that only one computation of the nonlinear dynamical problem is required. In Eq. (14), the function $g(t)$ is defined by

$$
g(t)=\frac{2 \pi \Delta \nu}{\pi} \operatorname{sinc}_{\pi}(t \Delta \nu) \cos (2 \pi s \Delta \nu t)
$$

where $x \mapsto \operatorname{sinc}_{\pi}(x)$ is the function defined by $\operatorname{sinc}_{\pi}(x)=\sin (\pi x) /(\pi x)$. Note that the Fourier transform of such function is

$$
\widehat{g}(2 \pi \nu)=\mathbb{1}_{\widetilde{\mathbb{B}}_{e}}(2 \pi \nu)
$$

in which $\mathbb{1}_{\widetilde{\mathbb{B}}}(x)=1$ if $x \in \mathbb{B}$ and 0 otherwise, and where $\widetilde{\mathbb{B}}_{e}=\left\{-\mathbb{B}_{\text {exc }}\right\} \bigcup\left\{\mathbb{B}_{\text {exc }}\right\}$ with

$$
\mathbb{B}_{\mathrm{exc}}=[2 \pi(s-1 / 2) \Delta \nu, 2 \pi(s+1 / 2) \Delta \nu] .
$$

It should be noted that such time-evolution excitation allows a forced-response problem and not a time-evolution problem with initial conditions to be considered. The forced-response problem is thus approximated by an equivalent time-evolution problem with zero initial conditions over a finite time interval, which includes almost all of the signal energy of the excitation.

\subsection{Uncertainty quantification induced by the mistuning}

The random nature of the mistuning is then considered by implementing the nonparametric probabilistic approach, which presents the capability to include both the system-parameter uncertainties and the model uncertainties induced by modeling errors (see [23] for a complete review on the subject). Since the analysis is carried out for the class of integrated bladed disks 
that are manufactured from a unique solid piece of material, the uncertainties are not considered to be independent from one blade to another one (in opposite to the case of a fan). The MEAN-NL-ROM is constructed by modal analysis without any sub-structuring techniques.

\subsubsection{Nonparametric probabilistic model for the mistuning}

It is assumed that only the linear operators of the structure are concerned with the mistuning phenomenon. The linear reduced operators $[\mathbf{m}],[\mathbf{d}],[\mathbf{c}(\Omega)],\left[\mathbf{k}^{(g)}\right],\left[\mathbf{k}^{(c)}(\Omega)\right]$, and $\left[\mathbf{k}^{(e)}\right]$ of the MEAN-NL-ROM are replaced by the random matrices $[\mathbf{M}],[\mathbf{D}],[\mathbf{C}(\Omega)],\left[\mathbf{K}^{(g)}\right],\left[\mathbf{K}^{(c)}(\Omega)\right]$, and $\left[\mathbf{K}^{(e)}\right]$ defined on the probability space $(\Theta, \mathcal{T}, \mathcal{P})$ such that $\mathrm{E}\{[\mathbf{M}]\}=[\mathbf{m}], \mathrm{E}\{[\mathbf{D}]\}=[\mathbf{d}]$, $\mathrm{E}\{[\mathbf{C}(\Omega)]\}=[\mathbf{c}(\Omega)], \mathrm{E}\left\{\left[\mathbf{K}^{(g)}\right]\right\}=\left[\mathbf{k}^{(g)}\right], \mathrm{E}\left\{\left[\mathbf{K}^{(c)}(\Omega)\right]\right\}=\left[\mathbf{k}^{(c)}(\Omega)\right]$, and $\mathrm{E}\left\{\left[\mathbf{K}^{(e)}\right]\right\}=\left[\mathbf{k}^{(e)}\right]$, in which $\mathrm{E}$ is the mathematical expectation.

Let $[\mathbf{a}]$ be a $(N \times N)$ matrix issued from the MEAN-NL-ROM with positive-definite property. For instance, it represents, the mass, the damping, the geometrical stiffness, the linear elastic stiffness or the centripetal stiffness. The corresponding random matrix $[\mathbf{A}]$ is then written as

$$
[\mathbf{A}]=\left[\mathbf{l}_{A}\right]^{\mathrm{T}}\left[\mathbf{G}_{A}\left(\delta_{A}\right)\right]\left[\mathbf{l}_{A}\right]
$$

in which $\left[\mathbf{I}_{A}\right]$ is the $(N \times N)$ upper triangular matrix issued from the Cholesky factorization of $[\mathbf{a}]$, and where $\left[\mathbf{G}_{A}\right]$ is a full random matrix with values in the set of all the positive-definite symmetric $(N \times N)$ matrices.

When $[\mathbf{a}]$ is the gyroscopic coupling matrix, the corresponding random matrix $[\mathbf{A}]$ is then written as

$$
[\mathbf{A}]=\left[\mathbf{t}_{A}\right]\left[\mathbf{l}_{A}\right]^{\mathrm{T}}\left[\mathbf{G}_{A}\left(\delta_{A}\right)\right]\left[\mathbf{l}_{A}\right],
$$

in which the matrices $\left[\mathbf{t}_{A}\right]$ and $\left[\mathbf{l}_{A}\right]$ are the $(N \times N)$ matrices defined by $\left[\mathbf{l}_{A}\right]=\left[\mathbf{s v}_{A}\right]^{1 / 2}\left[\mathbf{b}_{A}\right]^{\mathrm{T}}$ and $\left[\mathbf{t}_{A}\right]=[\mathbf{a}]\left[\mathbf{b}_{A}\right]\left[\mathbf{s v}_{A}\right]\left[\mathbf{b}_{A}\right]^{\mathrm{T}}$, in which the $(N \times N)$ full matrix $\left[\mathbf{b}_{A}\right]$ is constituted of the eigenvectors of $[\mathbf{a}][\mathbf{a}]^{\mathrm{T}}$ and where the $(N \times N)$ diagonal matrix $\left[\mathbf{s v}_{A}\right]$ is the singular values, issued from the single value decomposition (SVD) of matrix $[\mathbf{a}]$. Note that the probability distribution and the random generator of $\left[\mathbf{G}_{A}\left(\delta_{A}\right)\right]$ is detailed in [23]. The dispersion of random matrix $\left[\mathbf{G}_{A}\right]$ is controlled by the hyperparameter $\delta_{A}$ belonging an admissible set $\boldsymbol{\Delta}$. Consequently, the mistuning level of the bladed-disk is entirely controlled by the $\mathbb{R}^{6}$-valued hyperparameter $\delta=\left(\delta_{M}, \delta_{D}, \delta_{C}, \delta_{K_{g}}, \delta_{K_{c}}, \delta_{K}\right)$, belonging to the admissible set $\boldsymbol{\Delta}^{6}$. 


\subsubsection{STOCH-NL-ROM of the mistuned bladed-disk}

For constructing the STOCH-NL-ROM, the deterministic matrices in Eq. (3) are replaced by the random matrices. The deterministic displacement field $\mathbf{u}^{N}(\cdot, t)$ becomes a random field $\mathbf{U}^{N}(\cdot, t)$ that is written, for all $\mathbf{x} \in \Omega$, as

$$
\mathbf{U}^{N}(\mathbf{x}, t)=\sum_{\beta=1}^{N} \boldsymbol{\varphi}^{\beta}(\mathbf{x}) Q_{\beta}(t)
$$

in which the $\mathbb{R}^{N}$-valued random variable $\mathbf{Q}(t)=\left(Q_{1}(t), \cdots, Q_{N}(t)\right)$ is solution of the following set of stochastic nonlinear differential equations,

$$
M_{\alpha \beta} \ddot{Q}_{\beta}+\left(D_{\alpha \beta}+C(\Omega)_{\alpha \beta}\right) \dot{Q}_{\beta}+\left(K_{\alpha \beta}^{(e)}+K^{(c)}(\Omega)_{\alpha \beta}+K_{\alpha \beta}^{(g)}\right) Q_{\beta}+K_{\alpha \beta \gamma}^{(2)} Q_{\beta} Q_{\gamma}+K_{\alpha \beta \gamma \delta}^{(3)} Q_{\beta} Q_{\gamma} Q_{\delta}=f_{\alpha} .
$$

\subsubsection{Numerical aspects for solving the STOCH-NL-ROM}

The solution of the STOCH-NL-ROM is calculated using the Monte Carlo numerical simulation. For each realization $\theta$ belonging to $\Theta$, the set of $N$ deterministic nonlinear coupled differential equations is considered and solved with an implicit and unconditionally stable integration scheme (Newmark method with the averaging acceleration scheme). Introducing the notation $\mathbf{Q}_{i}(\theta)=\mathbf{Q}\left(t_{i} ; \theta\right)$, related to each sample time $t_{i}$, the following set of $N$ deterministic nonlinear equations is solved for computing $\mathbf{Q}_{i}(\theta)$

$$
\left[\mathbf{K}_{i}^{\mathrm{eff}}(\theta)\right] \mathbf{Q}_{i}(\theta)+\mathbf{F}^{\mathrm{NL}}\left(\mathbf{Q}_{i}(\theta)\right)=\mathbf{F}_{i}^{\mathrm{eff}}(\theta),
$$

in which the effective $(N \times N)$ matrix $\left[\mathbf{K}_{i}^{\text {eff }}(\theta)\right]$ and the effective force vector $\mathbf{F}_{i}^{\text {eff }}(\theta)$ are easily computed at each time $t_{i}$. Note that matrix $\left[\mathbf{K}_{i}^{\text {eff }}(\theta)\right]$ has no particular signature due to the presence of the gyroscopic coupling matrix. The nonlinear term $\mathbf{F}^{\mathrm{NL}}\left(\mathbf{Q}_{i}(\theta)\right)$, issued from the presence of the geometric nonlinearities, is written as

$$
F_{\alpha}^{\mathrm{NL}}\left(\mathbf{Q}_{i}(\theta)\right)=K_{\alpha \beta \gamma}^{(2)} Q_{i_{\beta}}(\theta) Q_{i_{\gamma}}(\theta)+K_{\alpha \beta \gamma \delta}^{(3)} Q_{i_{\beta}}(\theta) Q_{i_{\gamma}}(\theta) Q_{i_{\delta}}(\theta)
$$

For each sampling time $t_{i}$, the computation of solution $\mathbf{Q}_{i}(\theta)$ is addressed by the fixed point method because this iterative scheme is few time consuming and does not require the evaluation of the tangential matrix. Nevertheless, when the algorithm does not converge, it is replaced 
by the Crisfield arc-length method [31]. Such algorithm introduces a new additional scalar parameter $\Pi_{i}$ that multiplies the right-hand side member of the nonlinear equation. In this case, at each sampling time $t_{i}$, this nonlinear equation is written as

$$
\left[\mathbf{K}_{i}^{\mathrm{eff}}(\theta)\right] \mathbf{Q}_{i}(\theta)+\mathbf{F}^{\mathrm{NL}}\left(\mathbf{Q}_{i}(\theta)\right)=\Pi_{i}(\theta) \mathbf{F}_{i}^{\mathrm{eff}}(\theta)
$$

The nonlinear equation is solved step by step, each incremental step being characterized by a given arc length. For a given step, an iterative scheme requiring one evaluation of the tangential matrix allows a solution $\left(\mathbf{Q}_{i}(\theta), \Pi_{i}(\theta)\right)$ to be computed. Note that the tangential matrix can algebraically evaluated from Eq. (23). An adaptive arc length, depending on the number of iterations necessary to obtain the convergence of the preceding increment is also implemented according to [32] in order to accelerate the computation. An unusual procedure is then added to the algorithm, because Eq. (22) has to be solved instead of Eq. (24). In the nonlinear dynamical context, parameter $\Pi_{i}$ is deterministic and has to be controlled in order to reach the value 1 . This implies that the state of the algorithm corresponding to the preceding increment has to be stored. When $\Pi_{i}(\theta)$ is found to be upper than 1 , the algorithm is rewind to the preceding increment and the computation is set again with the half of the arc-length. Such additional procedure is repeated until parameter $\Pi_{i}(\theta)$ reaches 1 within a numerical tolerance set to $10^{-6}$. Even if such procedure is time consuming, because of the necessary evaluations of the tangential matrix and due to the procedure controlling the value of parameter $\Pi_{i}$, its main advantage is its capability of capturing high-nonlinear mechanical behaviors.

\section{Application to an industrial bladed-disk}

\subsection{Nonlinear deterministic analysis}

\subsubsection{Description of the external load}

The structure under consideration in an industrial centrifugal compressor belonging to the class of integrated bladed disks. Due to proprietary reasons, the number $M$ of blades characterizing the order of the cyclic symmetry of the structure cannot given. The finite element model of the structure is constructed with solid finite elements and is constituted of about 2, 000, 000 degrees of freedom. Fig. 1 displays a part of the finite element mesh of the investigated bladed disk. The structure is in rotation around its revolution axis with a constant velocity $\Omega=30,750 \mathrm{rpm}$. 
Since the dynamic analysis is carried out in the rotating frame of the structure, the rigid body motion due to the rotation of the structure corresponds to a fixed boundary condition at the inner radius of the structure. The bladed disk is made up of a homogeneous isotropic material. A modal damping model is added for the bladed disk.

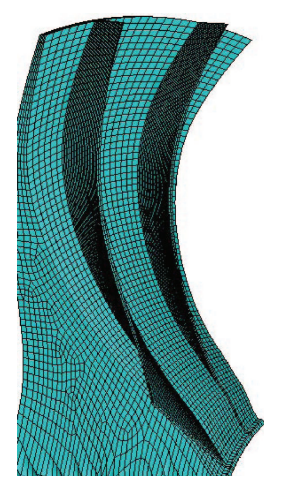

Figure 1: Finite element mesh of a part of the structure

The cyclic symmetry is first used for constructing the reduced matrices of the mean linear reduced-order model. The linear generalized eigenvalue problem related to the tuned bladeddisk is then solved using this cyclic symmetry property $[25,26]$. Let $\nu_{0}$ be the first eigenfrequency. Figure 2 displays the dimensionless eigenfrequencies $\nu_{i} / \nu_{0}$ of the tuned structure with respect to the circumferential wave number $h$. The graph is truncated to $h=5$ because only a 5 - th engine-order excitation is needed to be considered in the present industrial application. Two dimensionless frequency excitation bands $\mathbb{B}_{\mathrm{exc}}^{1}=[0.97,1.10]$ and $\mathbb{B}_{\mathrm{exc}}^{2}=[1.78,2.34]$ are investigated. Frequency excitation band $\mathbb{B}_{\mathrm{exc}}^{1}$ contains an insulated resonance whereas frequency band $\mathbb{B}_{\mathrm{exc}}^{2}$ contains a veering that is known to intensify the important effects of linear mistuning yielding the dynamical response to be usually amplified.

The excitation frequency bands are defined by parameters $s$ and $\Delta \nu$ given in Table 1 , for which the time domain function $g(t)$ is defined by Eq. (15) The initial instant of integration, $t_{i n i}$, and the total time duration, $T$, are summarized in Table 1. Concerning the spatial distribution of the load, the point excitations are distributed at the tip of each blade along the rotational axis and verify the cyclic geometry. The intensity $f_{0}$ of the external load is located in the range $\left.] 0,6\right] \mathrm{N}$, which induces a range of small to large amplitudes of the responses corresponding to a sweep from a linear response until a very high rate of geometrical nonlinearities. A high load intensity can be viewed as a situation for which the total damping of the bladed disk structure would 




Figure 2: Natural frequencies with respect to circumferential wave number

Table 1: Numerical parameters related to the excitation

\begin{tabular}{|l||c|c|c|c|}
\hline & $s$ & $\Delta \nu / \nu_{0}$ & $\nu_{0} t_{\text {ini }}$ & $\nu_{0} T$ \\
\hline \hline $\mathbb{B}_{\text {exc }}^{1}$ & 8 & 0.13 & -46.23 & 184 \\
\hline $\mathbb{B}_{\text {exc }}^{2}$ & 4 & 0.51 & -11.79 & 184 \\
\hline
\end{tabular}

reach very small values due to aeroelastic phenomenons. Such extreme situations are realistic when approaching flutter regimes. The frequency band of analysis is a broad frequency band corresponding to the dimensionless interval $\mathbb{B}=[0,3.34]$. The sample frequency is $\nu_{e} / \nu_{0}=$ 11.12, yielding the number $n_{t}$ of time steps to be $n_{t}=4,096$. The frequency resolution is then $\nu / \nu_{0},=0.0054$. Let $\widehat{g}(2 \pi \nu)$ be the Fourier transform of function $g(t)$. Figures 3 and 4 show the graphs $t / t_{0} \mapsto g(t)$ and $\nu / \nu_{0} \mapsto \widehat{g}(2 \pi \nu)$ for both cases corresponding to excitation frequency bands $\mathbb{B}_{\text {exc }}^{1}$ and $\mathbb{B}_{\text {exc }}^{2}$.

\subsubsection{Nonlinear tuned analysis}

For the construction of the MEAN-NL-ROM, the vector basis is chosen as explained in [21]. The nonlinear equations are solved in the subspace spanned by the usual linear basis constituted of the $N$ modal shapes related to the first $N$ increasing natural eigenfrequencies. A convergence analysis is carried out by increasing the value $N$ of the reduced-order model. It can be shown that $N=65$ yields a reasonable convergence, corresponding to $\nu_{N} / \nu_{0}=3.34$ From now on, 

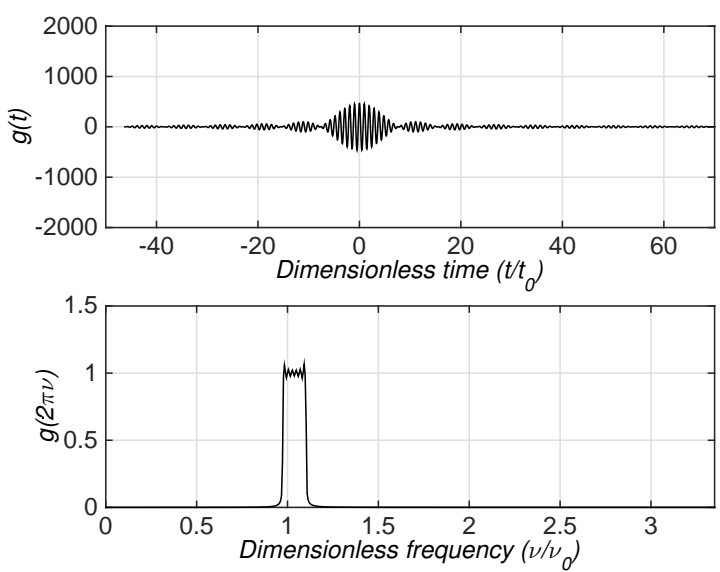

Figure 3: Representation of the external load in the time domain and in the frequency domain: graph of $t / t_{0} \mapsto g(t)$ (upper graph) and $\nu / \nu_{0} \mapsto \widehat{g}(2 \pi \nu)$ (lower graph) for $\mathbb{B}_{\text {exc }}^{1}=[0.97,1.10]$.
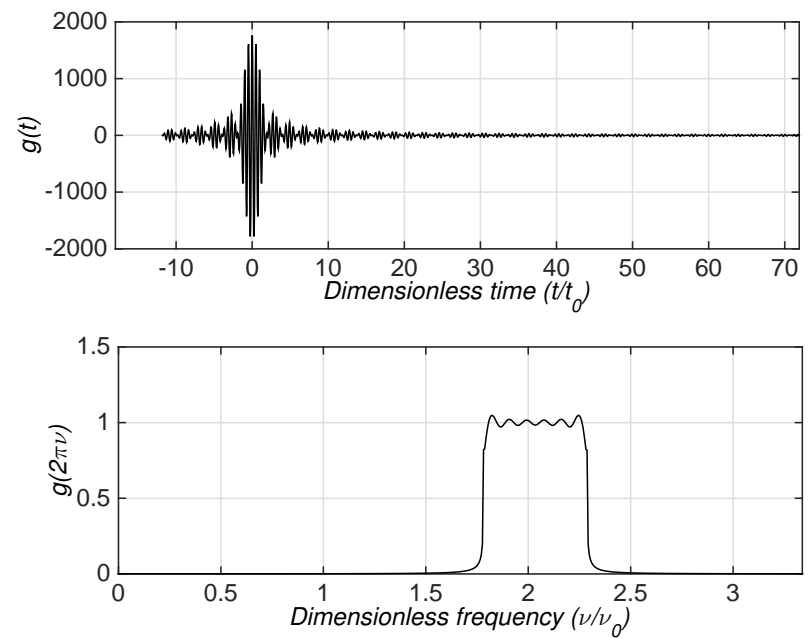

Figure 4: Representation of the external load in the time domain and in the frequency domain: graph of $t / t_{0} \mapsto g(t)$ (upper graph) and $\nu / \nu_{0} \mapsto \widehat{g}(2 \pi \nu)$ (lower graph) for $\mathbb{B}_{\text {exc }}^{2}=[1.78,2.34]$.

the converged solution corresponding to the observation issued from the MEAN-NL-ROM is denoted by $\widetilde{\mathbf{u}}(t)$. For clarity, when confusion is possible, superscripts L and NL will be added for distinguishing the linear case from the geometric nonlinear one. The two excitation cases are successively investigated.

Nonlinear tuned analysis related to excitation frequency band $\mathbb{B}_{\text {exc }}^{1}$

Being interested in the blade yielding the highest vibration amplitude, let $j_{0}$ be defined as $j_{0}=\arg \max _{j}\left(\max _{t} \widetilde{u}_{j}^{\mathrm{NL}}(t)\right)$. The observation $v(t)$ corresponding to the selected blade out- 
plane displacement is defined by $v(t)=\widetilde{u}_{j_{0}}(t)$. Figure 5 displays the graph $t / t_{0} \mapsto v^{\mathrm{L}}(t)$ (upper graph) and $t / t_{0} \mapsto v^{\mathrm{NL}}(t)$ (lower graph), corresponding to a load intensity $f_{0}=2.5 \mathrm{~N}$. Since the dynamical response related to these two cases is sensitively different, it can be deduced that $f_{0}=2.5 \mathrm{~N}$ corresponds to a load intensity for which the geometric nonlinear effects are significant, yielding a blade softening characterized by a relative long duration amplification of the blade displacement with respect to the linear case. There are no reasons to think that the frequency contents of the blade response shape be especially different in view of the time domain nonlinear response.
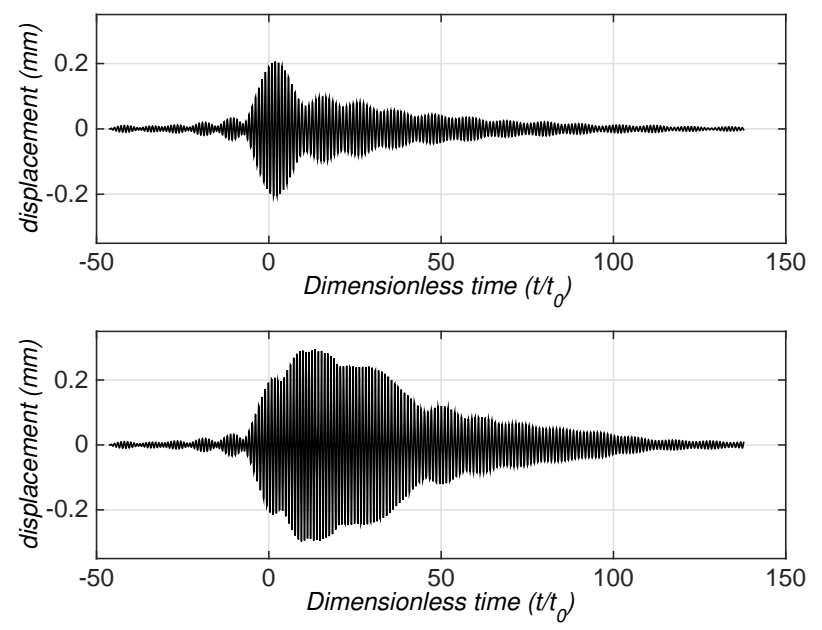

Figure 5: Time domain observation $t / t_{0} \mapsto v(t)$ related to the linear (upper graph) and the nonlinear (lower graph) cases for $\mathbb{B}_{\mathrm{exc}}^{1}=[0.97,1.10]$ and $f_{0}=2.5 \mathrm{~N}$.

Let $k_{0}=\arg \max _{j}\left(\max _{\nu / \nu_{0} \in \mathbb{B}} \widehat{\widetilde{u}}_{j}^{\mathrm{NL}}(2 \pi \nu)\right)$ for which $\widehat{\widetilde{u}}_{j}^{\mathrm{NL}}(2 \pi \nu)$ is the Fourier transform of $\widetilde{u}_{j}^{\mathrm{NL}}(t)$. In the frequency domain, the observation $w(2 \pi \nu)$ corresponding to the selected blade out-plane displacement is defined by $w(2 \pi \nu)=\widehat{\widetilde{u}}_{k_{0}}(2 \pi \nu)$. Figure 6 displays the graphs $\nu / \nu_{0} \mapsto w^{\mathrm{L}}(2 \pi \nu)$ (upper graph) and $\nu / \nu_{0} \mapsto w^{\mathrm{NL}}(2 \pi \nu)$ (lower graph). As expected for the linear case, it can be seen that the frequency content of the blade response coincides with $\mathbb{B}_{\mathrm{exc}}^{1}$. One can clearly see that there exist higher frequencies, which are located in dimensionless frequency band $[3,3.34]$ which are excited through the geometric nonlinearities. Nevertheless, its contribution is clearly negligible. Moreover, it is seen that the dimensionless eigenfrequencies corresponding to the three upper lines in Fig. 2, which are located in dimensionless frequency band $[1.5,2.8]$, are not excited by the energy transferred outside the frequency band of excita- 
tion (induced by the presence of geometrical nonlinearities).

Finally, a sensitivity analysis is conducted in order to quantify the geometric nonlinear effects with respect to the load intensity. Let $b_{w, \infty}$ and $b_{\nu, \infty}$ be the amplitude ratio and frequency ratio defined by

$$
\begin{aligned}
b_{w, \infty} & =\frac{\max _{\nu \in \mathbb{B}} w^{\mathrm{NL}}(2 \pi \nu)}{\max _{\nu \in \mathbb{B}} w^{\mathrm{L}}(2 \pi \nu)} \\
b_{\nu, \infty} & =\frac{\arg \max _{\nu \in \mathbb{B}} w^{\mathrm{NL}}(2 \pi \nu)}{\arg \max _{\nu \in \mathbb{B}} w^{\mathrm{L}}(2 \pi \nu)}
\end{aligned}
$$

Figure 7 displays the graphs $f_{0} \mapsto b_{w, \infty}\left(f_{0}\right)$ (upper graph) and $f_{0} \mapsto b_{\nu, \infty}\left(f_{0}\right)$ (lower graph). These graphs allow for showing if the analyzed response belongs or not to the nonlinear domain. When the values of observations $b_{w, \infty}$ and $b_{\nu, \infty}$ are different from 1 , the domain is nonlinear. It can be seen that some geometric nonlinear effects occur from $f_{0}=0.5 \mathrm{~N}$. A non-monotonic amplification (softening followed by hardening effects) at the main resonance peak, combined to a $10 \%$ shift towards the higher frequencies, is also observed.
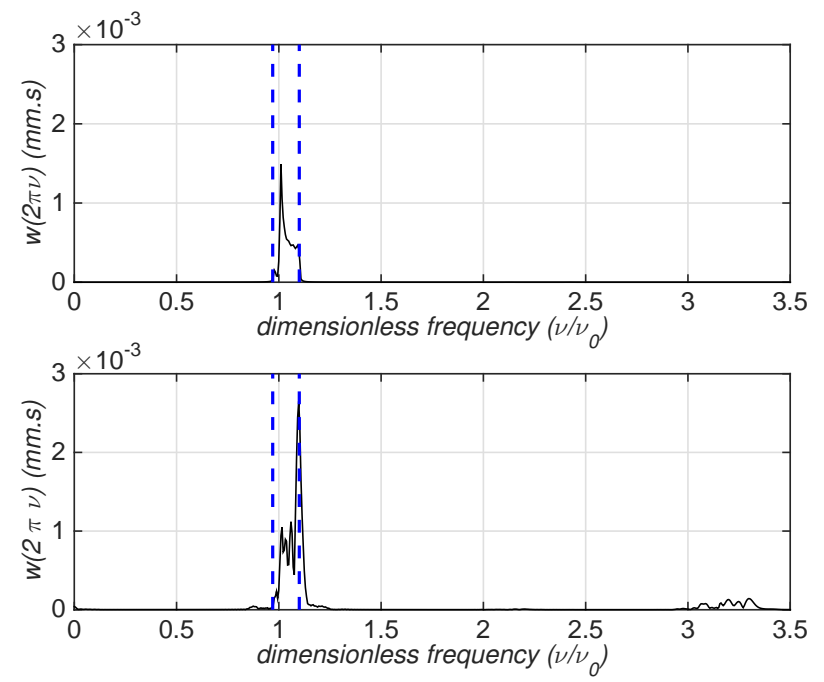

Figure 6: Frequency domain observation $\nu / \nu_{0} \mapsto w(2 \pi \nu)$ related to the linear (upper graph) and the nonlinear (lower graph) cases for $\mathbb{B}_{\mathrm{exc}}^{1}=[0.97,1.10]$ and $f_{0}=2.5 \mathrm{~N}$.

\section{Nonlinear tuned analysis related to excitation frequency band $\mathbb{B}_{\text {exc }}^{2}$}

Figure 8 displays the graph $t / t_{0} \mapsto v^{\mathrm{L}}(t)$ (upper graph) and $t / t_{0} \mapsto v^{\mathrm{NL}}(t)$ (lower graph), corresponding to a load intensity $f_{0}=2.5 \mathrm{~N}$. On these graphs, significant levels of geometrical nonlinear effects can be observed and the dynamical response is drastically different from the 

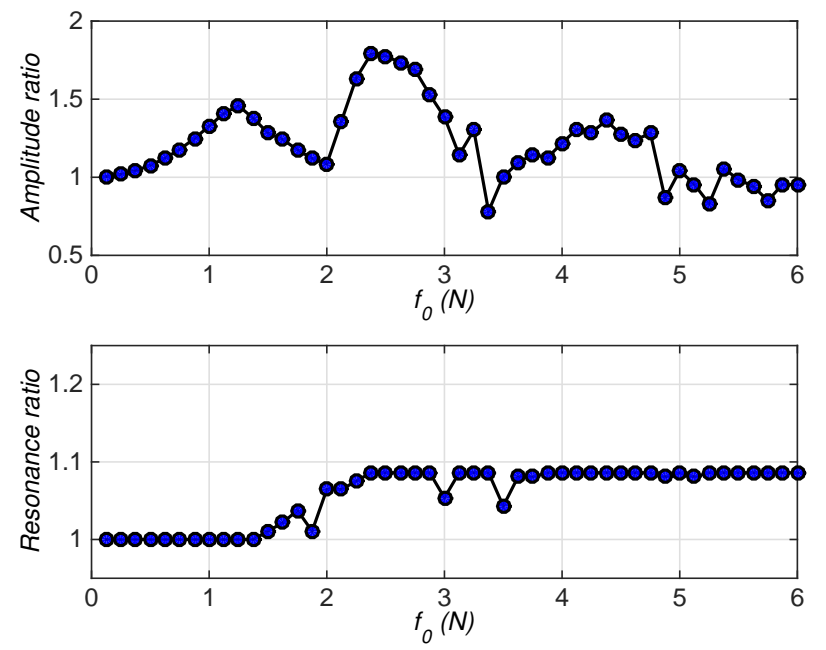

Figure 7: Sensitivity analysis with respect to the load intensity $f_{0}$.

previous case. With respect to the linear case, it can be seen that the geometric nonlinearities induce a blade stiffening characterized by a reduction of the vibration amplitudes of the blades from $2 \mathrm{~mm}$ until $1 \mathrm{~mm}$ This stiffening is also combined with a strong irregularity of the blade response shape in the time domain, which shows an enrichment of the frequency content that has to be quantified.
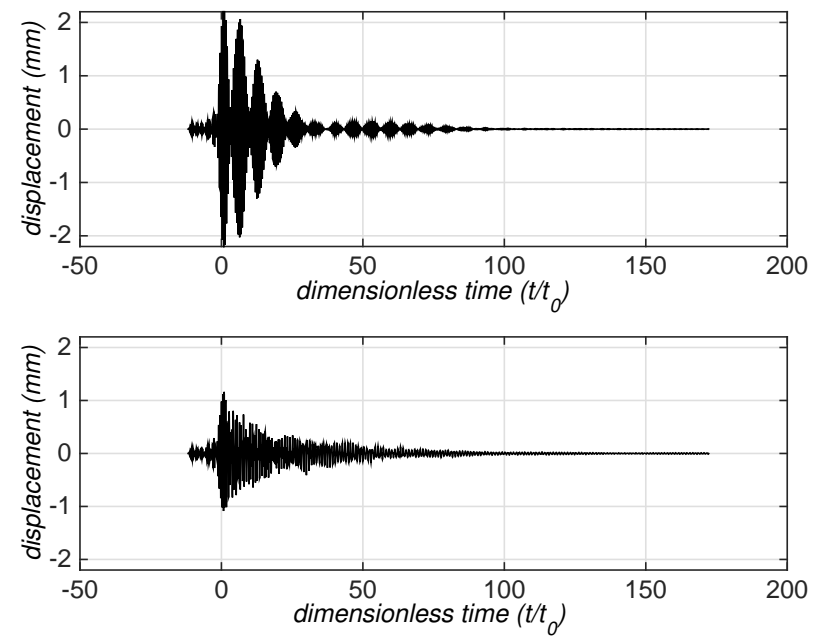

Figure 8: Time domain observation $t / t_{0} \mapsto v(t)$ related to the linear (upper graph) and the nonlinear (lower graph) cases for $\mathbb{B}_{\mathrm{exc}}^{2}=[1.78,2.34]$ and $f_{0}=2.5 \mathrm{~N}$.

Figure 9 displays the graphs $\nu / \nu_{0} \mapsto w^{\mathrm{L}}(2 \pi \nu)$ (upper graph) and $\nu / \nu_{0} \mapsto w^{\mathrm{NL}}(2 \pi \nu)$ (lower graph). The spreading of the vibrational energy over the whole frequency band of analysis 
$\mathbb{B}$ is due to the nonlinear geometric effects and is characterized through secondary response peaks. Again, for high frequencies that are located outside $\mathbb{B}_{\mathrm{exc}}^{2}$ in dimensionless frequency band $[3,3.34]$, the dynamical response induced by the geometric nonlinearities is negligible. Nevertheless, some new resonances appear with the same order of magnitude than the main resonance in the dimensionless frequency band $[1,1.5]$. We put then in evidence a complex dynamical behavior that can be dangerous because non-expected resonances with non-negligible amplitudes appear outside excitation frequency band $\mathbb{B}_{\text {exc }}^{2}$.
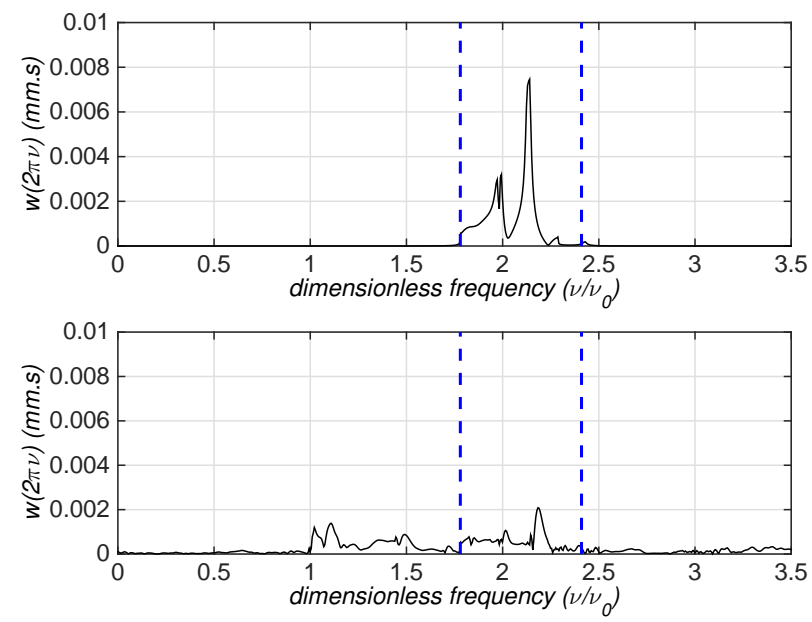

Figure 9: Frequency domain observation $\nu / \nu_{0} \mapsto w(2 \pi \nu)$ related to the linear (upper graph) and the nonlinear (lower graph) cases for $\mathbb{B}_{\text {exc }}^{2}=[1.78,2.34]$ and $f_{0}=2.5 \mathrm{~N}$.

It should be noted that the nonlinear dynamical behavior is different according to the choice of the frequency band of excitation. It is clearly seen that the geometric nonlinearities excite harmonics which are outside the frequency band of excitation and mainly for frequencies lower than the ones located in the excitation band. This can explain why the mechanical energy remains concentrated in $\mathbb{B}_{\text {exc }}^{1}$ with a local amplification. On the contrary, there are many eigenfrequencies below frequency band $\mathbb{B}_{\text {exc }}^{2}$ of excitation, which are excited through the geometric nonlinearities. As a consequence, the energy transfer to these very low eigenfrequencies is consequent, yielding a consequent spread of energy and amplitude ratios below 1 .

Figure 10 displays the graphs $f_{0} \mapsto b_{w, \infty}\left(f_{0}\right)$ (upper graph) and $f_{0} \mapsto b_{\nu, \infty}\left(f_{0}\right)$ (lower graph), which is very different from the previous case. A quick decreasing in the amplitude ratio of the main resonance located in frequency band $\mathbb{B}$ is observed with respect to the load intensity. 
More precisely, we detect that the critical intensity load from which the geometric nonlinearities affect the dynamical response is $f_{0}=0.375 N$. Concerning the resonance ratio, we can see a slight shift of the resonance frequency to higher frequencies for intensity loads lower than $f_{0}=2.5 \mathrm{~N}$. Nevertheless, an irregular behavior is put in evidence for $f_{0} \geq 2.75 \mathrm{~N}$. The resonance ratio suddenly falls down to very low values of resonance ratio belonging to $[0.5,0.8]$. It can be shown an irregular alternating between small values of the resonance ratio and the values around 1 of the resonance ratio. Such behavior means that there exist situations for which some resonances occur due to geometric nonlinearities, and which correspond to subharmonics that become the most important. Such an observation gives rise to a worrying dynamical behavior that points out the necessity of taking into account the geometric nonlinearities in the computational model.
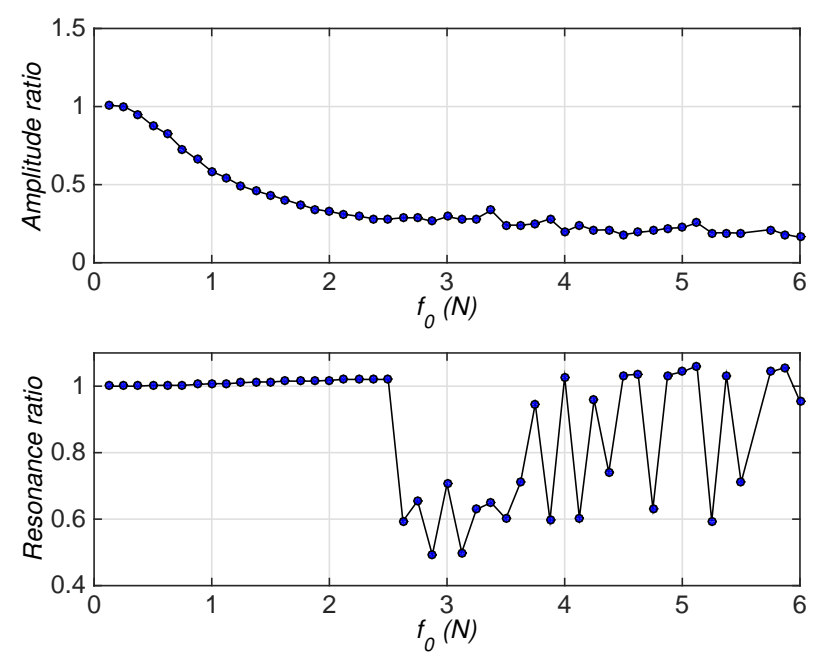

Figure 10: Sensitivity analysis with respect to load intensity $f_{0}$.

Figure 11 displays the graph $t / t_{0} \mapsto v^{\mathrm{L}}(t)$ (upper graph) and $t / t_{0} \mapsto v^{\mathrm{NL}}(t)$ (lower graph), corresponding to a load intensity $f_{0}=2.75 \mathrm{~N}$. No noticeable differences can be seen between the time-domain nonlinear responses of Figs. 8 and 11, which only differ by a slight difference of load intensity. Nevertheless, comparing these nonlinear responses in the frequency domain yield significant differences. Figure 12 displays the graphs $\nu / \nu_{0} \mapsto w^{\mathrm{L}}(2 \pi \nu)$ (upper graph) and $\nu / \nu_{0} \mapsto w^{\mathrm{NL}}(2 \pi \nu)$ (lower graph). It can be seen that the main resonance amplitude is nearly twice the resonance amplitude located in $\mathbb{B}_{\mathrm{exc}}^{2}$. Moreover, a broad range of frequency band $[1,1.5]$ is excited, yielding a large number of resonances with the same order of magnitude 
than the resonance amplitudes located in $\mathbb{B}_{\text {avs. }}^{2}$.
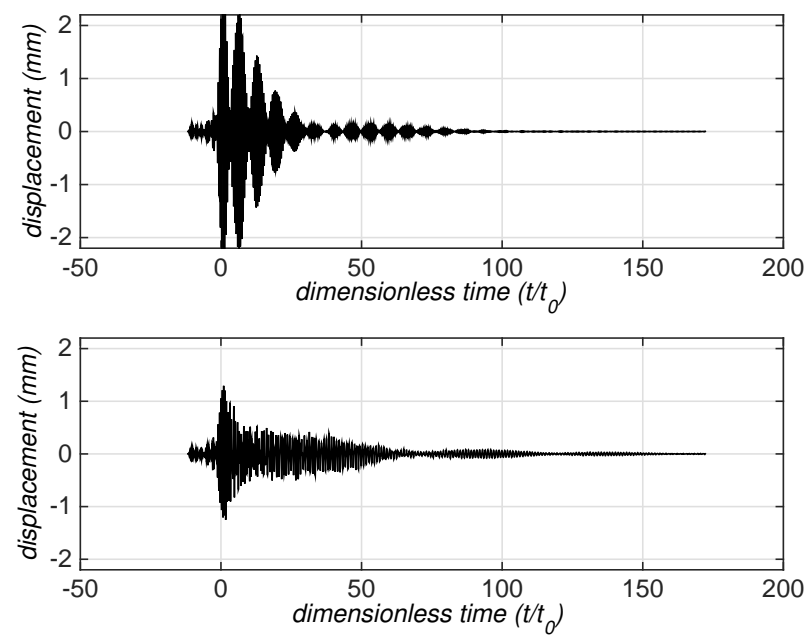

Figure 11: Time domain observation $t / t_{0} \mapsto v(t)$ related to the linear (upper graph) and the nonlinear (lower graph) cases for $\mathbb{B}_{\mathrm{exc}}^{2}=[1.78,2.34]$ and $f_{0}=2.75 \mathrm{~N}$.
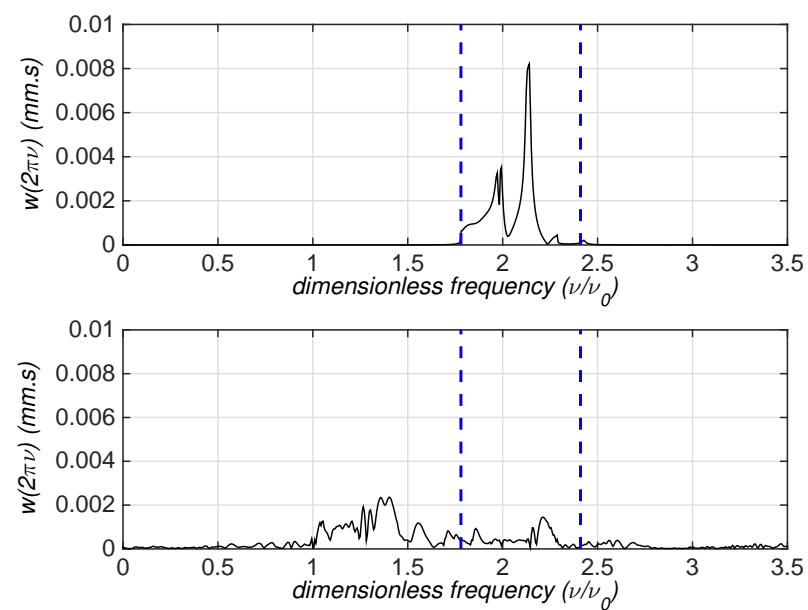

Figure 12: Frequency domain observation $\nu / \nu_{0} \mapsto w(2 \pi \nu)$ related to the linear (upper graph) and the nonlinear (lower graph) cases for $\mathbb{B}_{\text {exc }}^{2}=[1.78,2.34]$ and $f_{0}=2.75 \mathrm{~N}$.

\subsection{Nonlinear analysis for the mistuned bladed disk}

In the present case, the MEAN-NL-ROM is constructed using the linear eigenmodes of vibrations as projection basis. Note that no substructuring methods have been used. This means that the uncertainties are not considered as independent from one blade to another one, which is coherent with the structure under consideration belonging to the class of integrated bladed 
disks, that are manufactured from a unique solid piece of metal. In the present analysis, for a better understanding of the phenomenon, only the matrices related to the linear part are random. The mistuning level is thus controlled by the $\mathbb{R}^{5}$-vector $\delta=\left(\delta_{M}, \delta_{D}, \delta_{C}, \delta_{K_{c}}, \delta_{K}\right)$.

\subsubsection{Sensitivity analysis according to the type of uncertainties}

The mistuning analysis is carried out in the frequency domain, which then requires a Fourier transform of the observation. First, a sensitivity analysis is conducted in order to identify the dispersion parameters yielding the most significant effects on the mistuned response represented by the random variable $W(2 \pi \nu)$ corresponding to observation $w(2 \pi \nu)$ in the tuned case.

As expected for the linear mistuned case, it can be observed that an uncertainty level of 0.1 for the gyroscopic coupling, or/and the centrifugal stiffness or/and the damping terms, has a very limited impact on the linear mistuned response, for excitation band $\mathbb{B}_{\mathrm{exc}}^{1}$ or $\mathbb{B}_{\mathrm{exc}}^{2}$. Figures 13 and 14 compare the confidence region of observation $W^{\mathrm{NL}}(2 \pi \nu)$ with a probability level set to 0.95 , when the frequency excitation band is $\mathbb{B}_{\mathrm{exc}}^{1}$ or $\mathbb{B}_{\mathrm{exc}}^{2}$. In the first case, the nonlinear response seems to be insensitive to uncertainties on the whole band of analysis $\mathbb{B}$. In the second case, the nonlinear mistuned behavior is shown to be substantially different.On one hand, the nonlinear mistuned response behaves almost like its tuned response in the part of the frequency band corresponding to the frequency band of excitation $\mathbb{B}_{\text {exc }}^{2}$. On the other hand, the uncertainties spread because of the geometrical nonlinearities, yielding large confidence regions for the nonlinear mistuned response in $\mathbb{B} \backslash \mathbb{B}_{\text {exc }}^{2}$, more particularly in the dimensionless range $[1,1.2]$. The response in this range shows that some realizations have amplification levels around 2 while the mistuned response remains almost unchanged in $\mathbb{B}_{\text {exc }}^{2}$. Note that a similar nonlinear mistuned behavior can be observed for the cases for which centrifugal stiffness or damping are uncertain. Figures 15 and 17 (resp. Figs. 16 and 18) display the graphs of the confidence region of observation $W^{\mathrm{L}}(2 \pi \nu)$ and $W^{\mathrm{NL}}(2 \pi \nu)$ with a probability level set to 0.95 for a mass uncertainty level $\delta_{M}=0.1$ and a load intensity $f_{0}=2.5 N$ in the excitation band $\mathbb{B}_{\text {exc }}^{1}$ (resp. $\mathbb{B}_{\text {exc }}^{2}$ ). By comparing Figs. 15 and 16, it can be seen for the usual linear mistuned case, that the random response of the blade is particularly sensitive to uncertainties yielding important amplification response levels. For the case corresponding to an excitation located in $\mathbb{B}_{\text {exc }}^{1}$, the presence of geometric nonlinearities drastically improve the robustness of the random response in $\mathbb{B}_{\mathrm{exc}}^{1}$, since the upper confidence region envelope of the confidence region does not exceed the tuned resonance. 


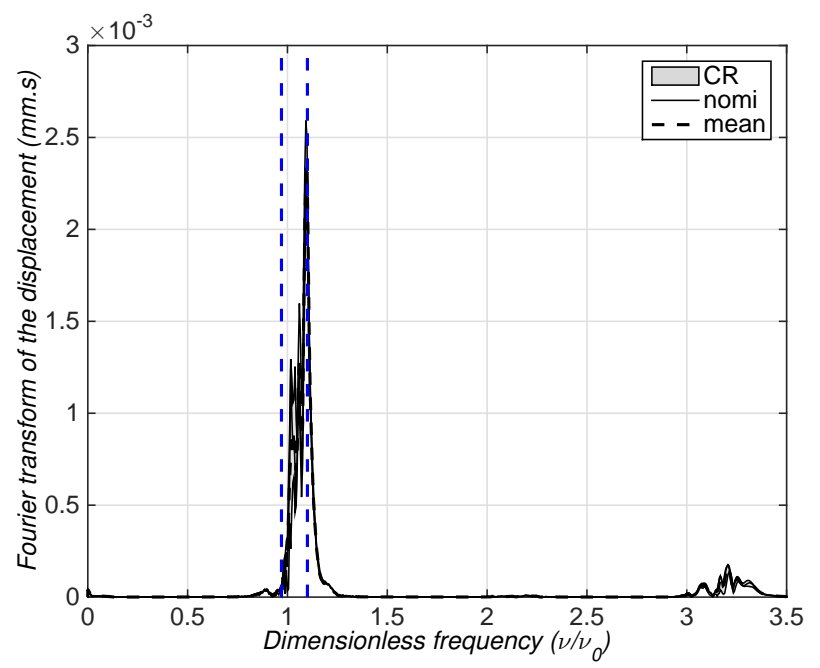

Figure 13: Stochastic analysis: frequency domain observation related to the nonlinear case for $\delta_{C}=0.1$ and for excitation frequency band $\mathbb{B}_{\text {exc }}^{1}$ : mean model (thick line), mean of the stochastic model (thin dashed line), confidence region (gray region).

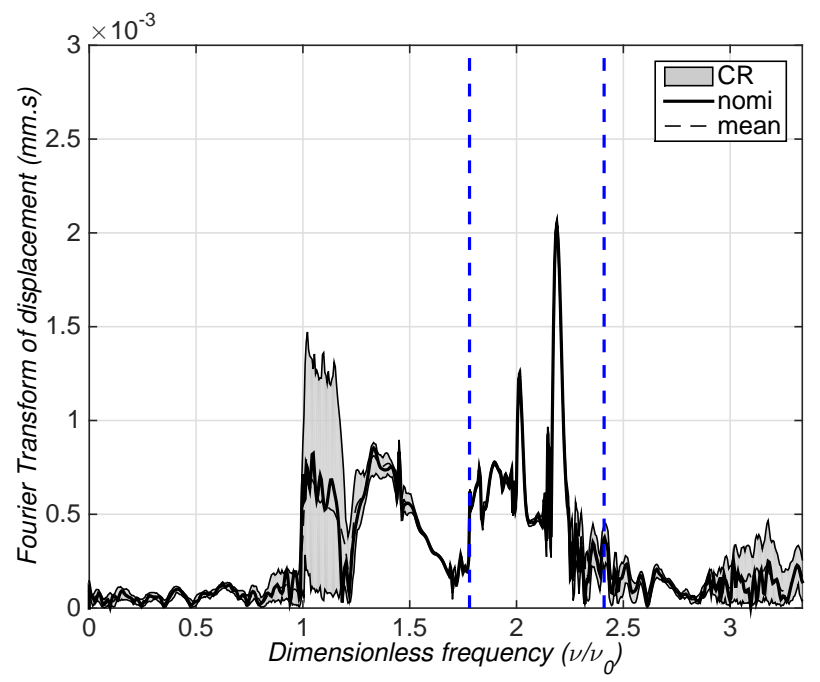

Figure 14: Stochastic analysis: frequency domain observation related to the nonlinear case for $\delta_{C}=0.1$ and for excitation frequency band $\mathbb{B}_{\text {exc }}^{2}$ : mean model (thick line), mean of the stochastic model (thin dashed line), confidence region (gray region).

Moreover, the response level outside $\mathbb{B}_{\text {exc }}^{1}$ is one order magnitude smaller, and the presence of uncertainties does not affect the nonlinear dynamical behavior of the blades.

By comparing Figs. 17 and 18, it can be seen again that the presence of he geometric nonlinearities tends to inhibit the nonlinear dynamical amplification induced by the mistuning in the 


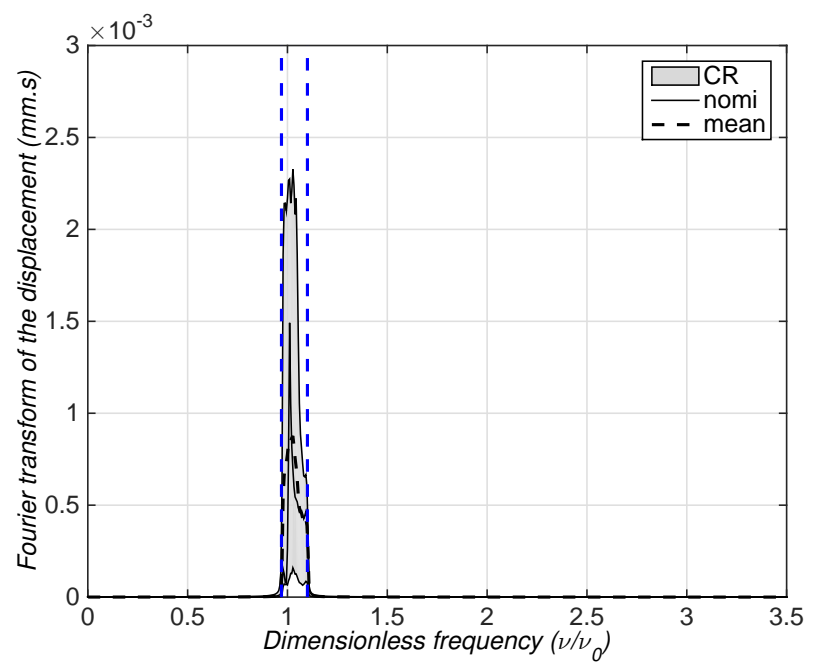

Figure 15: Stochastic analysis: frequency domain observation related to the linear case for $\delta_{M}=0.1$ and for excitation frequency band $\mathbb{B}_{\text {exc }}^{1}$ : mean model (thick line), mean of the stochastic model (thin dashed line), confidence region (gray region).

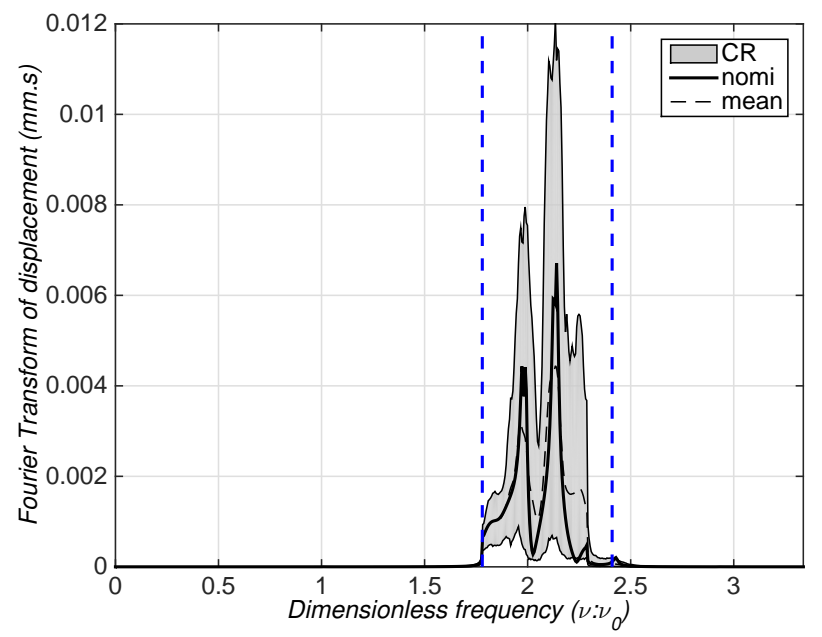

Figure 16: Stochastic analysis: frequency domain observation related to the linear case for $\delta_{M}=0.1$ and for excitation frequency band $\mathbb{B}_{\text {exc }}^{2}$ : mean model (thick line), mean of the stochastic model (thin dashed line), confidence region (gray region).

frequency band $\mathbb{B}_{\text {exc }}^{2}$ corresponding to the excitation frequency band, limiting the dynamical amplification level to a magnitude 1.2. Moreover, a widespread of uncertainties is observed in $\mathbb{B} \backslash \mathbb{B}_{\text {exc }}^{2}$ with dynamic amplification levels of magnitude 2.5 when performing the nonlinear dynamic analysis in subfrequency band $\mathbb{B}_{\text {sub }}=[1,1.6]$. In this band, it can be seen a nonlinear mistuned response level similar to the nonlinear tuned response located in $\mathbb{B}_{\mathrm{exc}}^{2}$. Note that a 




Figure 17: Stochastic analysis: frequency dependent observation related to the nonlinear case for $\delta_{M}=0.1$ and for excitation frequency band $\mathbb{B}_{\text {exc }}^{1}$ : mean model (thick line), mean of the stochastic model (thin dashed line), confidence region (gray region).

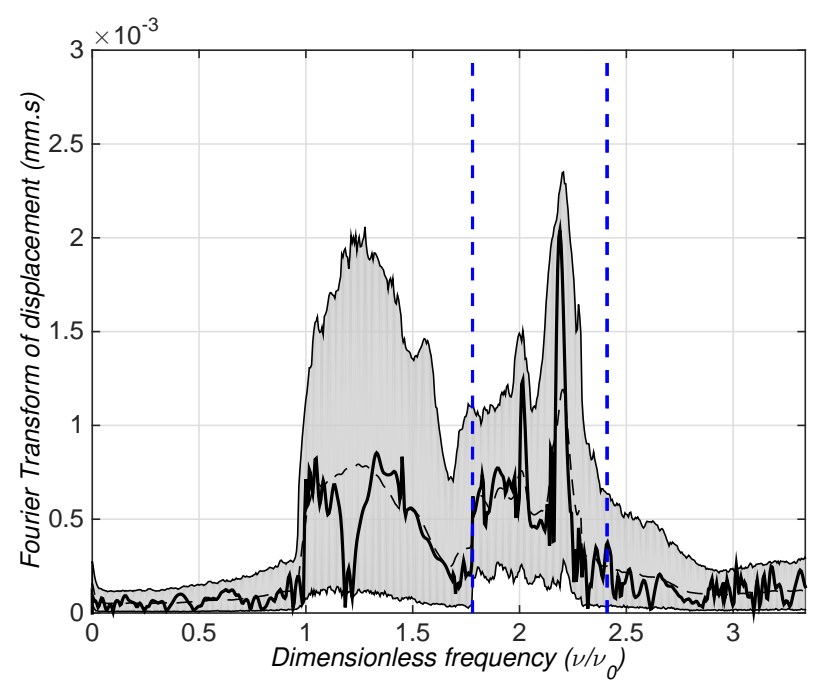

Figure 18: Stochastic analysis: frequency dependent observation related to the nonlinear case for $\delta_{M}=0.1$ and for excitation frequency band $\mathbb{B}_{\mathrm{exc}}^{2}$ : mean model (thick line), mean of the stochastic model (thin dashed line), confidence region (gray region).

similar behavior is observed for the case of elastic-stiffness uncertainties.

\subsubsection{Nonlinear dynamical analysis for the mistuned response}

The analysis is focussed for the excitation frequency band $\mathbb{B}_{\mathrm{exc}}^{2}$ that exhibits the complex dynamic situation described above. The load intensity is fixed to $f_{0}=2.5$ and the uncertainty 
level is set to $\boldsymbol{\delta}=\left(\delta_{M}, \delta_{D}, \delta_{C}, \delta_{K_{c}}, \delta_{K}\right)=(\delta, 0.2,0.2,0.2, \delta)$, in which $\delta$ is defined hereinafter. Thus, the effects of mass and elastic uncertainties combined to uncertainties for the rotational effects are taken into account in the analysis.

A sensitivity analysis according to parameter $\delta$ is carried out in order to establish a comparison of the mistuning effects between the linear and the nonlinear mistuned cases. For fixed $\nu / \nu_{0} \in$ $\mathbb{B}$, let $Y(2 \pi \nu)$ be the random dynamic amplification factor defined by

$$
Y(2 \pi \nu)=\frac{W(2 \pi \nu)}{\max _{\nu / \nu_{0} \in \mathbb{B}} w(2 \pi \nu)}
$$

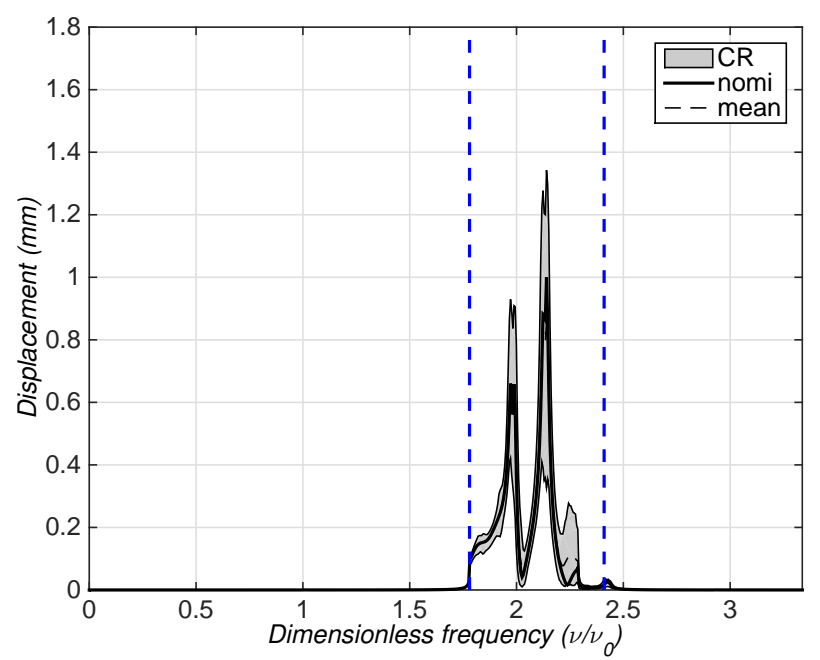

Figure 19: Stochastic analysis: frequency domain observation $Y^{\mathrm{L}}(2 \pi \nu)$ related to the linear case for $\delta_{K}=\delta_{M}=$ 0.02 and for $\delta_{K_{c}}=\delta_{C}=\delta_{D}=0.2$ : mean model (thick line), mean of the stochastic model (thin dashed line), confidence region (gray region).

Figures 19 (or 21) and Figs. 20 (or 22) show the confidence region of the linear and nonlinear observations $Y^{\mathrm{L}}(2 \pi \nu)$ and $Y^{\mathrm{NL}}(2 \pi \nu)$ for $\delta=0.02$ (or $\delta=0.16$ ). In frequency band $\mathbb{B}_{\mathrm{exc}}^{2}$, it can be observed that an increasing of the mistuning yields a uniform spread in the frequency domain around the main resonance, yielding a weak robustness with respect to uncertainties. It is also clearly seen that the linearized assumption tends to increase the extreme values of the response levels. The geometric nonlinear effects clearly inhibit the amplification of the random response. More particularly, the extreme values related to $Y^{\mathrm{NL}}(2 \pi \nu)$ yield moderate amplification even if the confidence region remains relatively broad.

For frequency band $\mathbb{B}_{\text {sub }}$ that is highly sensitive to uncertainties, it can be seen that an increasing of $\delta$ yields a small spread around the resonances located outside frequency band $\mathbb{B}_{\text {exc }}^{2}$, accompa- 


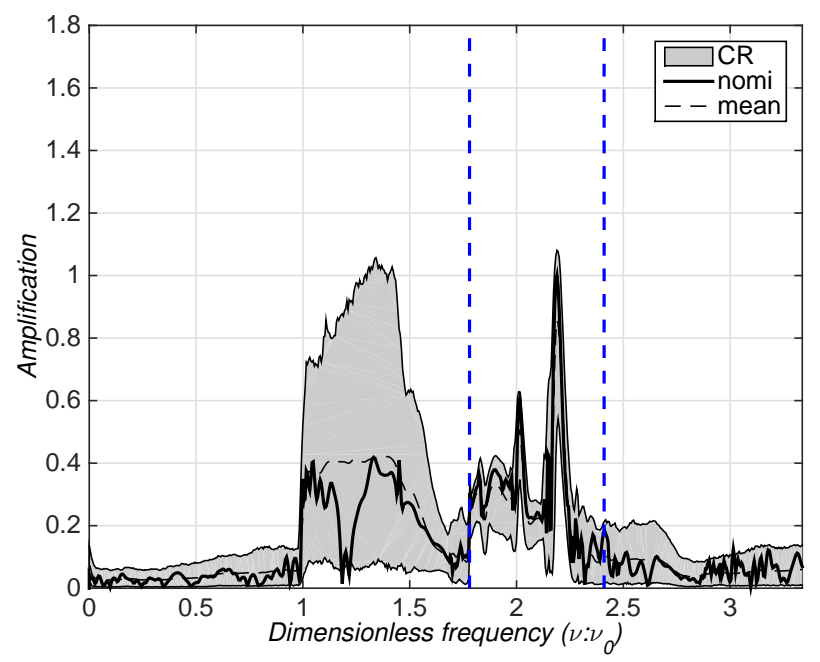

Figure 20: Stochastic analysis: frequency domain observation $Y^{\mathrm{NL}}(2 \pi \nu)$ related to the nonlinear case for $\delta_{K}=$ $\delta_{M}=0.02$ and for $\delta_{K_{c}}=\delta_{C}=\delta_{D}=0.2$ : mean model (thick line), mean of the stochastic model (thin dashed line), confidence region (gray region).

nied by a moderate amplification of the response. In summary, the geometric nonlinear effects seem to mainly act on the uncertainty propagation by spreading the response on the whole band of analysis without drastically amplifying the amplitudes of the resonances located in $\mathbb{B}_{\text {exc }}^{2}$. The consequences of such a result mainly concern subfrequency band $\mathbb{B}_{\text {sub }}$, for which the geometric nonlinearities act as an internal excitation, yielding the occurrence of additional resonances. The wide spread of uncertainties through a large bandwidth gives rise to important amplifications with respect to these resonances.

For the analysis, the chosen observation is a random amplification factor. Such observation characterizes the dynamic amplification over the given frequency band with respect to a reference level taken as the resonance level of the tuned nonlinear system. In this case, the frequency band is the whole frequency band of analysis. Let $Y_{\infty}$ be the random amplification factor defined by $Y_{\infty}=\max _{\nu / \nu_{0} \in \mathbb{B}} Y(2 \pi \nu)$. We then define the second random amplification factor, $Z_{\infty}$, such that

$$
Z_{\infty}=\frac{\max _{\nu / \nu_{0} \in \mathbb{B}_{\text {sub }}} W(2 \pi \nu)}{\max _{\nu / \nu_{0} \in \mathbb{B}_{\text {sub }}} w(2 \pi \nu)}
$$

For that case, the chosen frequency band does not contain the excitation band but only subharmonics. In that way, the reference level taken for calculating the amplification factor corresponds to the secondary resonance level localized outside the frequency band of excitation. 


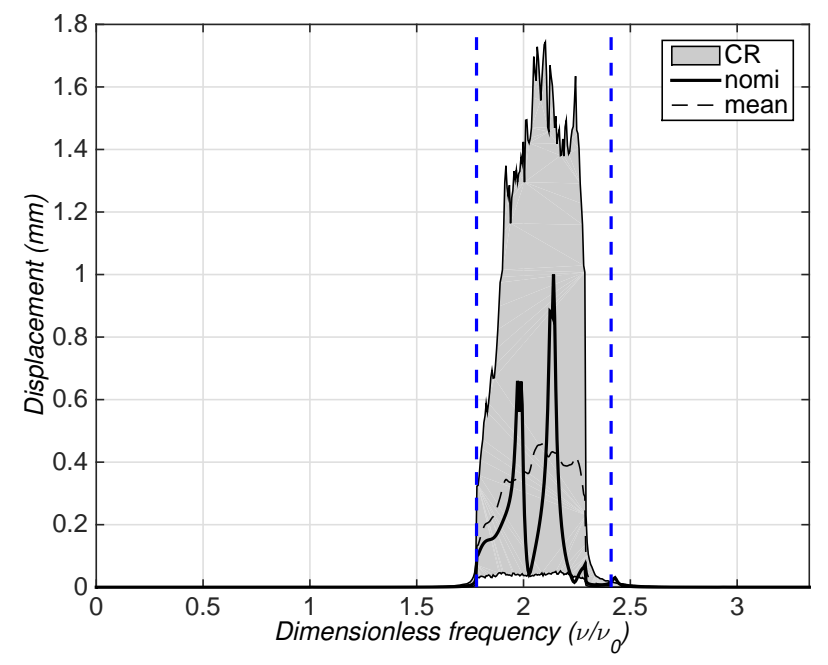

Figure 21: Stochastic analysis: frequency domain observation related to the linear case $Y^{\mathrm{L}}(2 \pi \nu)$ for $\delta_{K}=\delta_{M}=$ 0.16 and for $\delta_{K_{c}}=\delta_{C}=\delta_{D}=0.2$ : mean model (thick line), mean of the stochastic model (thin dashed line), confidence region (gray region).

Figures 23 displays the graph of the quantiles of $Y_{\infty}^{\mathrm{L}}$ with respect to mistuning rate $\delta$. As commonly observed, this graph displays a strong sensitivity of the random dynamic amplification with respect to uncertainties. Figure 24 compares the similar graphs obtained with random observations $Y_{\infty}^{\mathrm{NL}}$ and $Z_{\infty}^{\mathrm{NL}}$. Again, it is observed that the geometric nonlinear effects yield a limited sensitivity to mistuning uncertainties for observation $Y_{\infty}^{\mathrm{NL}}$. Figure 25 displays the probability density function of $Y_{\infty}^{\mathrm{NL}}$ for several values of $\delta$. It can clearly be seen that the probability density functions (pdf) exhibit a support that is $[0.55,1.5]$. These pdf are not symmetric, yielding amplification factors greater than 1 with a lower probability level. Furthermore, from $\delta \geq 0.23$, the shape of the pdfs seems to be less sensitive to the level of uncertainties. In Fig. 24, a special attention must be given to $Z_{\infty}^{\mathrm{NL}}$ that presents similar characteristics to $Y_{\infty}^{\mathrm{L}}$. In particular, these graphs exhibit a maximum, yielding the possibility to define some robustness areas that limit the dynamic amplification. It then points out, not only a complex sensitivity to uncertainties, but also high amplification levels that may yield unexpected amplifications. Figure 26 displays the pdfs of $Z_{\infty}^{\mathrm{NL}}$ for several values of $\delta$. These pdfs exhibit a support that is $[0.55,3.3]$. In particular, contrary to random observation $Y_{\infty}^{\mathrm{NL}}$, it can be shown that random observation $Z_{\infty}^{\mathrm{NL}}$ is particularly sensitive to $\delta$. It can also be noticed that these pdfs exhibit a slight bimodal 


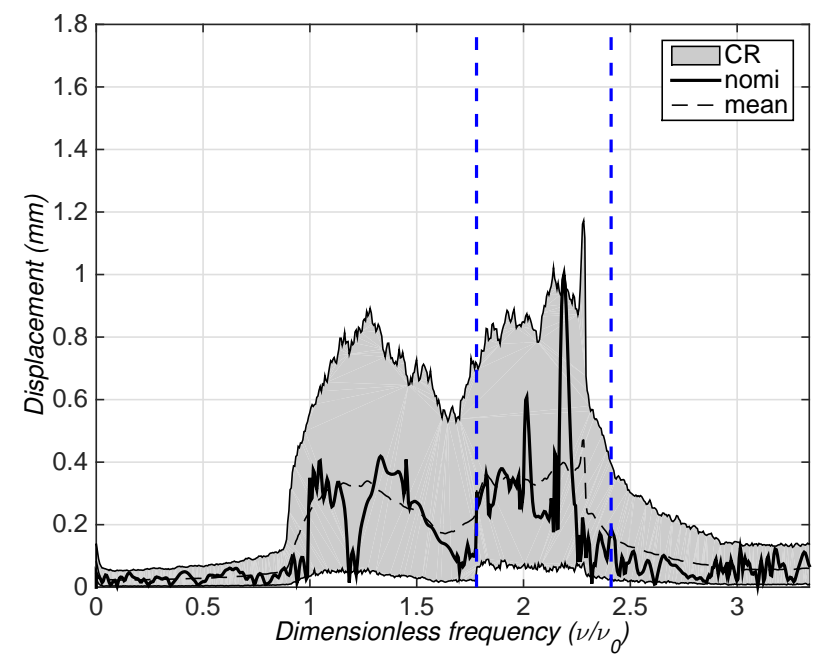

Figure 22: Stochastic analysis: frequency domain observation $Y^{\mathrm{NL}}(2 \pi \nu)$ related to the nonlinear case for $\delta_{K}=$ $\delta_{M}=0.16$ and for $\delta_{K_{c}}=\delta_{C}=\delta_{D}=0.2$ : mean model (thick line), mean of the stochastic model (thin dashed line), confidence region (gray region).

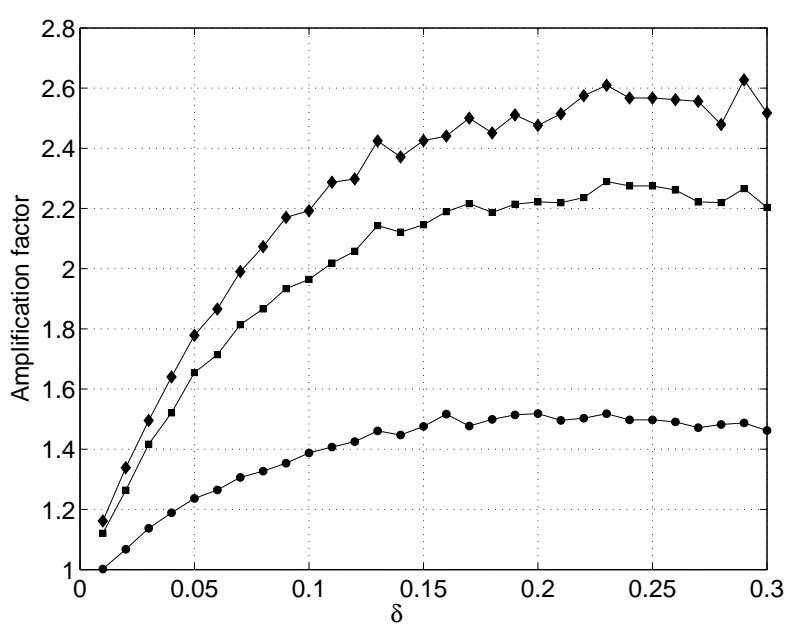

Figure 23: Quantile analysis of amplification factor $Y_{\infty}^{\mathrm{L}}$ with respect to mistuning level $\delta$ with 0.5 quantile (• symbol), 0.9 quantile $(\boldsymbol{\square}$ symbol), 0.95 quantile $(\diamond$ symbol $)$

behavior combined to a strong asymmetry with positive skewness.

\section{Conclusion}

A detailed dynamic analysis has been conducted in presence of uncertainties induced by mistuning in order to quantify the impact of the nonlinear geometrical effects on the mistuned 

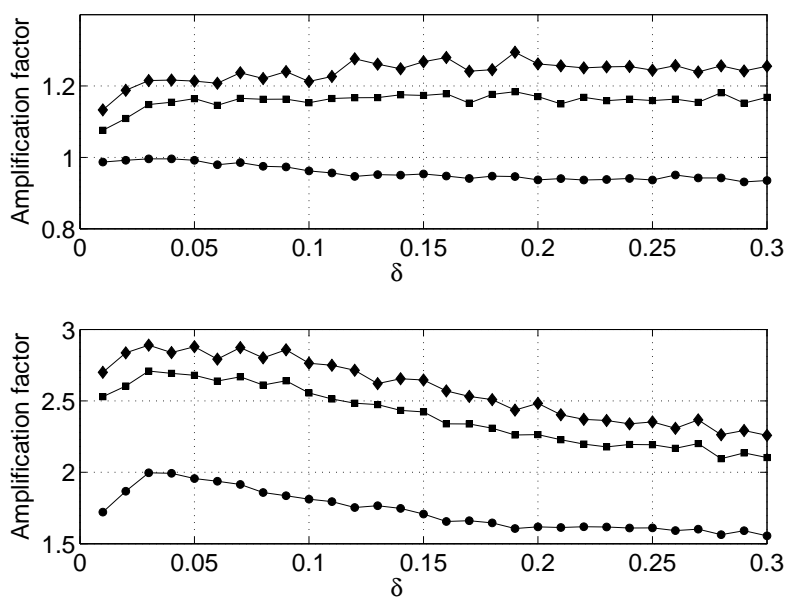

Figure 24: Quantile analysis of amplification factors $Y_{\infty}^{\mathrm{NL}}$ (upper graph) and $Z_{\infty}^{\mathrm{NL}}$ (lower graph) with respect to mistuning level $\delta$ with 0.5 quantile $(\bullet$ symbol), 0.9 quantile $(\boldsymbol{\square}$ symbol), 0.95 quantile $(\diamond$ symbol)
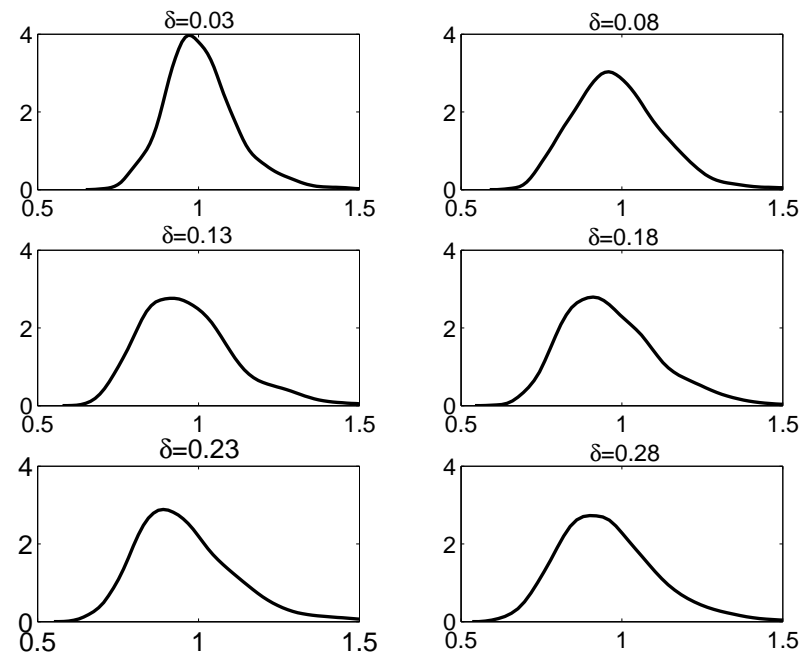

Figure 25: Stochastic analysis: probability density function of $Y_{\infty}^{\mathrm{NL}}$ for $\delta=0.03,0.08,0.13,0.18,0.23,0.28$.

structure, in terms of dynamic amplification of the responses and of uncertainty propagation.

In order to achieve such a detailed analysis, an advanced methodology has been applied to an industrial bladed disk for performing a mistuning analysis of bladed disks in the context of high amplitude loads inducing strong geometric nonlinear effects. One of the main goal of the work was to quantify those geometric nonlinear effects on the random dynamical response of the uncertain mistuned structure. On this basis, two load cases have been investigated, that correspond, for the usual linear mistuned case, to two situations that are known to yield either 

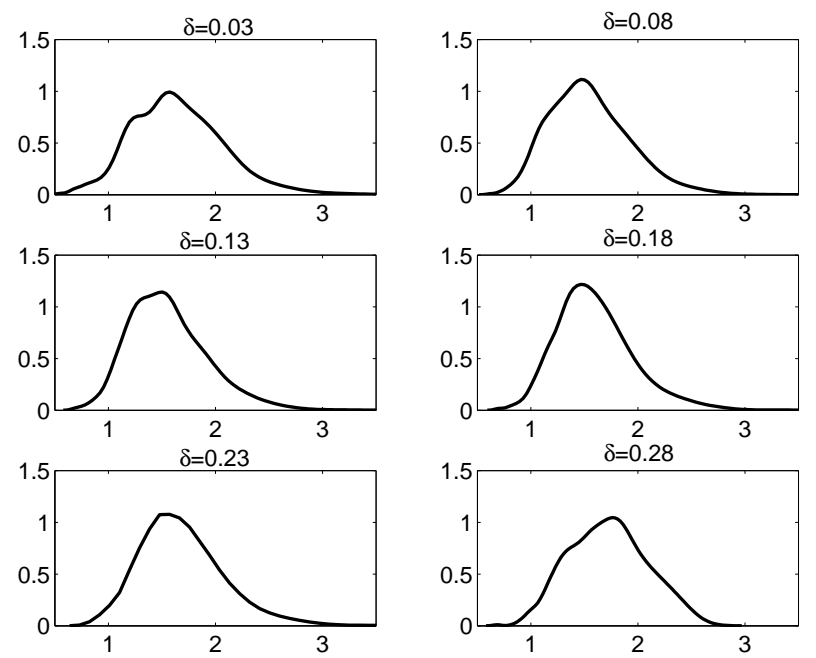

Figure 26: Stochastic analysis: probability density function of $Z_{\infty}^{\mathrm{NL}}$ for $\delta=0.03,0.08,0.13,0.18,0.23,0.28$.

a moderate response or either a response with a large dynamical amplification. The numerical results presented display new complex dynamical behaviors of the dynamical response of the blades.

Concerning the nonlinear tuned response, in the very low-frequency range and for an excitation corresponding to pure blade modes, the response exhibits higher amplitude levels, while mainly remaining located in the frequency band of excitation. In the opposite, in the low-frequency range, when the excitation is located around a veering, characterizing a strong coupling between the blade modes and the disk modes, a sudden decreasing of the vibration motion is observed and the nonlinear tuned response is spread outside the frequency band of excitation, yielding secondary resonances corresponding to sub-harmonics whose contribution cannot be longer neglected. In this case, a sensitivity analysis with respect to the load level exhibits a load threshold, from which a chaotic behavior is observed. Thus, the main resonance is randomly located in the frequency band of excitation or in the sub-harmonic range.

Considering the nonlinear mistuned response with uncertainties, the geometric nonlinear effects play an important role for the propagation of uncertainties. In particular, the robustness of the random response with respect to uncertainties remains strong in the frequency band of excitation, yielding reasonable amplification levels. However, such robustness suddenly falls in the sub-harmonic frequency range giving rise to consequent local amplification levels.

In summary, all the numerical results have demonstrated that the life duration of the indus- 
trial bladed disk can be very sensitive to the presence of geometric nonlinearities combined with mistuning effects. A careful attention has to be paid with the numerical modeling of the bladed-disk mistuning when dealing with exceptional operating regimes for which geometric nonlinearities have to be taken into account.

\section{Acknowledgements}

This work was supported by the DGA (French defence procurement agency) in the context of the TURBODYNA project (project number ANR-13-ASTR-0008-01) related to the ANR ASTRID research program (specific support scheme for research works and innovation defence). SAFRAN Turbomeca is also acknowledged for giving permission to publish this work.

\section{References}

[1] S.-T. Wei, C. Pierre, Localization phenomena in mistuned assemblies with cyclic symmetry part ii: Forced vibrations, ASME Journal of Vibration, Acoustics, Stress, and Reliability in Design 110 (4) (1988) 439-449.

[2] R. Bladh, M. Castanier, C. Pierre, Component-mode-based reduced order modeling techniques for mistuned bladed disks-part 1: Theoretical models, ASME Journal of Engineering for Gas Turbines and Power 123 (1) (2001) 89-99.

[3] E. Capiez-Lernout, C. Soize, Nonparametric modeling of random uncertainties for dynamic response of mistuned bladed-disks., ASME Journal of Engineering for Gas Turbines and Power 126 (3) (2004) 610-618.

[4] A. Rivas-Guerra, M. Mignolet, J. Delor, Identification of mistuning characteristics of bladed disks from free response data (part 2), ASME Journal of Engineering for Gas Turbines and Power 123 (2) (2001) 404-411.

[5] M. Castanier, C. Pierre, Using intentional mistuning in the design of turbomachinery rotors, AIAA Journal 40 (10) (2002) 2077-2086.

[6] B.-K. Choi, J. Lentz, R.-G. A.J., M.-P. Mignolet, Optimization of intentional mistuning patterns for the reduction of the forced response effects of unintentional mistuning: 
Formulation and assessment, ASME Journal of Engineering for Gas Turbines and Power 125 (1) (2003) 131-140.

[7] Y.-J. Chan, D.-J. Ewins, Management of the variability of vibration response levels in mistuned bladed discs using robust design concept. part 1: Parameter design., Mechanical Systems and Signal Processing 24 (8) (2010) 2777-2791.

[8] M. Mbaye, C. Soize, J.-P. Ousty, E. Capiez-Lernout, Robust analysis of design in vibration of turbomachines, ASME Journal of Turbomachinery 135 (2) (2013) 021008-1-8.

[9] D. Laxalde, F. Thouverez, J.-J. Sinou, J.-P. Lombard, Qualitative analysis of forced response of blisks with friction ring dampers", European Journal of Mechanics, A/Solids 26 (4) (2007) 676-687.

[10] E.-P. Petrov, Analysis of sensitivity and robustness of forced response for nonlinear dynamic structures, Mechanical Systems and Signal Processing 23 (1) (2009) 68-86.

[11] E.-P. Petrov, Analysis of flutter-induced limit cycle oscillations in gas-turbine structures with friction, gap, and other nonlinear contact interfaces, ASME Journal of Turbomachinery 134 (6) (2012) 061018-1-13.

[12] A. Muryavov, S. Rizzi, Determination of nonlinear stiffness with application to random vibration of geometrically nonlinear structures, Computers \& Structures 81 (2003) 15131523.

[13] M.-P. Mignolet, C. Soize, Stochastic reduced order models for uncertain geometrically nonlinear dynamical systems, Computer Methods in Applied Mechanics and Engineering 197 (2008) 3951-3963.

[14] A. Vakakis, Dynamics of a nonlinear periodic structure with cyclic symmetry, ASME Journal of Turbomachinery 95 (1-4) (1992) 197-226.

[15] E. Sarrouy, A. Grolet, F. Thouverez, Global and bifurcation analysis of a structure with cyclic symmetry, International Journal of Nonlinear Mechanics 46 (5) (2011) 727-737. 
[16] A. Grolet, F. Thouverez, Vibration analysis of a nonlinear system with cyclic symmetry, ASME Journal of Engineering for Gas Turbines and Power 133 (2) (2011) 022502-1-9.

[17] A. Grolet, F. Thouverez, Free and forced vibration analysis of a nonlinear system with cyclic symmetry: Application to a simplified model, Journal of Sound and Vibration 331 (12) (2012) 2911-2928.

[18] L. Sirovich, Turbulence and the dynamics of coherent structures , Quarterly of Applied Mathematics 45 (3) (1987) 561-571.

[19] E. Capiez-Lernout, C. Soize, M.-P. Mignolet, Post-buckling nonlinear static and dynamical analyses of uncertain cylindrical shells and experimental validation, Computer Methods in Applied Mechanics and Engineering 271 (2014) 210-230.

[20] M.-P. Mignolet, A. Przekop, S. Rizzi, S. Spottswood, A review of indirect/non-intrusive reduced order modeling of nonlinear geometric structures, Journal of Sound and Vibration 332 (10) (2013) 2437-2460.

[21] E. Capiez-Lernout, C. Soize, M.-P. Mignolet, Computational stochastic statics of an uncertain curved structure with geometrical nonlinearity in three-dimensional elasticity., Computational Mechanics 49 (1) (2012) 87-97.

[22] C. Touze, M. Vidrascu, D. Chapelle, Direct finite element computation of non-linear modal coupling coefficients for reduced-order shell models, Computational Mechanics 54 (2) (2014) 567-580.

[23] C. Soize, Stochastic Models of Uncertainties in Computational Mechanics, Lecture Notes in Engineering Mechanics 2, American Society of Civil Engineers (ASCE), 2012.

[24] C. Desceliers, C. Soize, S. Cambier, Non-parametric - parametric model for random uncertainties in nonlinear structural dynamics: Application to earthquake engineering., Earthquake Engineering and Structural Dynamics 33 (2003) 315-327.

[25] D. Thomas, Dynamics of rotationnaly periodic structures, International Journal for Numerical Methods in Engineering 14 (1979) 81-102. 
[26] A. Bossavit, Boundary value problems with symmetry and their approximation by finite elements, SIAM Journal of Applied Mathematics 53 (5) (1993) 1352-1380.

[27] L. Wagner, J. Griffin, A continuous analog model for grouped-blade vibration, Journal of Sound and Vibration 165 (1993) 421-438.

[28] J. Kenyon, J. Griffin, N. Kim, Sensitivity of tuned bladed disk response to frequency veering., ASME Journal of Engineering for Gas Turbines and Power 127 (4) (2005) 835842.

[29] P. Bisegna, G. Caruso, Dynamical behavior of disordered rotationally periodic structures: A homogenization approach., Journal of Sound and Vibration 330 (2011) 2608-2627.

[30] Y.-J. Chan, D.-J. Ewins, The amplification of vibration response levels of mistuned bladed disks: Its consequences and its distribution in specific situations, ASME Journal of Engineering for Gas Turbines and Power 133 (10) (2011) 102502-1-8.

[31] M. Crisfield, Non-linear finite element analysis of solids and structures, Vol. 1 : essentials, John Wiley and Sons, Chichester, 1997.

[32] R. de Borst, M. Crisfield, J. Remmers, C. Verhoosel, Non-linear finite element analysis of solids and structures, Wiley, 2012. 